\title{
Indole-Based Hydrazones Containing A Sulfonamide Moiety as Selective Inhibitors of Tumor-Associated Human Carbonic Anhydrase Isoforms IX and XII
}

\author{
Kübra Demir-Yazıcı ${ }^{1}$, Silvia Bua ${ }^{2}(\mathbb{D})$, Nurgül Mutlu Akgüness ${ }^{1}$, Atilla Akdemir ${ }^{3}{ }^{(D)}$, \\ Claudiu T. Supuran ${ }^{2}$ (1) and Özlen Güzel-Akdemir ${ }^{1, *}$ \\ 1 Department of Pharmaceutical Chemistry, Faculty of Pharmacy, Istanbul University, 34116 Istanbul, Turkey; \\ kubra.demir@istanbul.edu.tr (K.D.-Y.); akgunes.nurgul@gmail.com (N.M.A.) \\ 2 Department of NEUROFARBA, Sezione di Scienze Farmaceutiche Universita degli Studi di Firenze, \\ Sesto Fiorentino, 50019 Florence, Italy; silvia.bua@unifi.it (S.B.); claudiu.supuran@unifi.it (C.T.S.) \\ 3 Computer-Aided Drug Discovery Laboratory, Department of Pharmacology, Faculty of Pharmacy, \\ Bezmialem Vakif University, 34093 Istanbul, Turkey; aakdemir@bezmialem.edu.tr \\ * Correspondence: oguzel@istanbul.edu.tr
}

Received: 1 April 2019; Accepted: 10 May 2019; Published: 12 May 2019

\begin{abstract}
Novel sulfonamidoindole-based hydrazones with a 2-(hydrazinocarbonyl)-3-phenyl-1Hindole-5-sulfonamide scaffold were synthesized and tested in enzyme inhibition assays against the tumor-associated carbonic anhydrase isoforms, hCA IX and XII, and the off-targets, hCA I and II. The compounds showed selectivity against hCA IX and XII over hCA I and II. Six compounds showed $K_{\mathrm{I}}$ values lower than $10 \mathrm{nM}$ against hCA IX or XII. Molecular modeling studies were performed to suggest binding interactions between the ligand and the hCA active sites.
\end{abstract}

Keywords: carbonic anhydrase; sulfonamides; hydrazones; enzyme inhibition; molecular modeling

\section{Introduction}

Carbonic anhydrases (CA) are metalloenzymes that catalyze the reversible hydration of $\mathrm{CO}_{2}$ to $\mathrm{HCO}_{3}{ }^{-}$and $\mathrm{H}^{+}$. This simple but important reaction is widespread amongst many if not all organisms [1]. Many of the six structurally different classes of CAs (i.e., $\alpha, \beta, \gamma, \delta, \zeta$, and $\eta C A s$ ) contain a Zinc ion $\left(\mathrm{Zn}^{2+}\right)$ at the active site that is essential for catalysis [2]. CAs have several important physiological and pathological functions, such as respiration and transporting $\mathrm{CO}_{2} / \mathrm{HCO}_{3}{ }^{-}$between metabolizing tissues and lungs, electrolyte secretion [3], $\mathrm{pH}$ regulation [4], and biosynthetic reactions such as gluconeogenesis, lipogenesis, ureagenesis [5] and carcinogenicity [6].

The human carbonic anhydrases (hCAs) are a member of the $\alpha \mathrm{CAs}$, which are divided into 16 different isozymes (hCA I-XVI) [7]. These enzymes share similar structure, especially in the active site, but show different catalytic activities and tissue distributions [8]. Cytosolic hCA isoforms I and II (hCA I and hCA II) are widespread in the human body and are targets of clinically used diuretics [9], antiglaucoma [10], drugs, and anticonvulsants [11]. In contrast, transmembrane isoforms, hCA IX and hCA XII, which have an active site on the extracellular part of the cell membrane, are located mainly on hypoxic tumor cells [12] and are validated drug targets for the design of anticancer agents specific for solid hypoxic tumors [13]. Proliferation and survival of tumor cells seem to be closely related to overexpression of both enzymes [14]. Thus, selective inhibition of hCA IX and hCA XII with well characterized CA inhibitors (CAIs) may result in new drugs in chemotherapy.

Sulfonamides, a main class of CAIs, show their effect by binding to the $\mathrm{Zn}^{2+}$ ion of the hCA active site. Many compounds with a sulfonamide moiety are in clinical use as CAIs or at the development process. Most of the sulfonamides act as strong CAIs for many hCAs-including isoforms I and 
II-causing a wide range of side effects. However, the design of new CAIs, which show selective inhibition for tumor-associated isoenzymes, hCA IX and XII, and weak affinity for hCA I and II, currently receives great attention in medicinal chemistry research [15-17]. Indole based sulfonamide derivatives were investigated during the last years and have been evaluated as selective inhibitors of several classes of carbonic anhydrases, including human/mammalian isoforms and pathogens [18,19]. Members of our group investigated 2-(hydrazinocarbonyl)-3-phenyl- $1 H$-indole-5-sulfonamide for their interaction with 12 carbonic anhydrase isoforms in the search of compounds with good inhibitory activity against isozymes, such as CA I, II, VA, VB, VII, IX, and XII, among others [20]. Thus, we explore here the synthesis and structure-activity relationship (SAR) for the inhibition of four CA isoforms (hCA I, II, IX, and XII) with hydrazone derivatives of these compounds as putative CAIs.

\section{Results}

\subsection{Chemistry}

As was previously reported by our group, 3-phenyl-5-sulfonamido- $1 H$-indole-2-carbohydrazide 1 was prepared from sulfanilamide as outlined in Scheme 1. After diazotization of the sulfanilamide, condensation of diazonium salt with ethyl 2-benzylacetoacetate 2 led to an intermediate, which was cyclized in acidic medium with formation of the ethyl ester derivative of 3 , which was converted to 3 by treatment with hydrazine [20]. Further treatment of 3 with an appropriate carbonyl compound (ketone or aldehyde) yielded hydrazone derivatives 4-24 (Scheme 1).<smiles>CCOC(=O)C(Cc1ccccc1)C(C)=O</smiles>

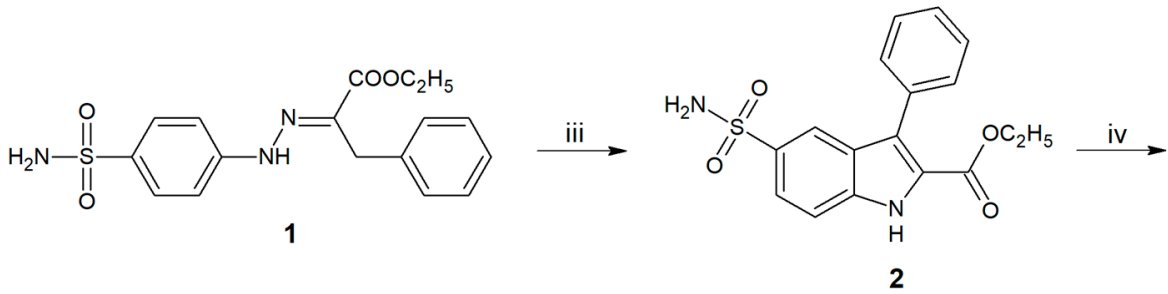<smiles>[R]C([R])=NNC(=O)c1[nH]c2ccc(S(N)(=O)=O)cc2c1-c1ccccc1</smiles>

Scheme 1. Reagents and conditions: (i) $\mathrm{NaNO}_{2}, \mathrm{HCl}, 0{ }^{\circ} \mathrm{C}$; (ii) $\mathrm{KOH}, 0{ }^{\circ} \mathrm{C}$; (iii) $37 \% \mathrm{HCl}$, reflux, 4 h; (iv) $\mathrm{H}_{2} \mathrm{NNH}_{2} \cdot \mathrm{H}_{2} \mathrm{O}, \mathrm{EtOH}$, reflux, $6 \mathrm{~h}$; (v) reflux, 6-12 h.

Newly synthesized hydrazones were characterized with melting points and spectral analyses. The IR spectra of compounds 4-24 showed NH stretching bands of the hydrazide-hydrazone group and sulfonamide moiety at the indole ring at $3423-3138 \mathrm{~cm}^{-1}$. The carbonyl functionalities of the new compounds were confirmed by strong $C=O$ stretching bands observed in the $1643-1685 \mathrm{~cm}^{-1}$, while compound 3 showed a band of $1627 \mathrm{~cm}^{-1}$, as expected. Asymmetric and symmetric $\mathrm{SO}_{2}$ stretching vibrations of the sulfonamide group had absorption bands in the $1344-1311 \mathrm{~cm}^{-1}$ and $1176-1147 \mathrm{~cm}^{-1}[21,22]$. 
The ${ }^{1} \mathrm{H}-\mathrm{NMR}$ spectrum of compounds displayed the $\mathrm{N}-\mathrm{H}$, the $\mathrm{C}_{4}-\mathrm{H}$, the $\mathrm{C}_{6}-\mathrm{H}$, and the $\mathrm{C}_{7}-\mathrm{H}$ protons of the indole ring at 12.39-12.14 ppm (for 9, the second indole ring $\mathrm{N}-\mathrm{H}$ proton was at $11.59 \mathrm{ppm}$ ) as a singlet and 8.85-7.15 ppm, 7.75-7.71, and 7.65-7.61 ppm, respectively. The N-H protons of the hydrazide structure exhibited the expected singlets at the 11.75-9.01 ppm [19]. The $\mathrm{SO}_{2} \mathrm{NH}_{2}$ protons had signals at 7.21-7.15 ppm. For compounds 4-11, the azomethine protons resonated at 9.24-8.09 ppm except 10. A broad resonance with $2 \mathrm{H}$ integration value at 8.21 and $8.24 \mathrm{ppm}$ was assigned to the two azomethine protons of $\mathbf{1 0}$. All the other protons were observed in the expected regions. In the ${ }^{13} \mathrm{C}$ NMR spectrum of compounds, carbon atoms of the hydrazide carbonyl (CONH) and the hydrazone $(C=N)$ groups were observed at in the order of 167.18-157.75 and 157.50-143.19 ppm.

\subsection{Enzyme Inhibition Assays}

All synthesized compounds were tested in enzyme inhibition assays against the widespread and cytosolic hCA I and II enzymes and the tumor-associated and transmembrane hCA IX and XII enzymes with extracellular catalytic domains (Table 1, Figure 1). The compounds showed selectivity for hCA IX and XII over hCA I and II (Table 1, Figure 1). For hCA IX, 14 of the tested compounds had $K_{I}$ values smaller than $100 \mathrm{nM}$, including one compound with a $K_{\mathbf{I}}$ value of less than $10 \mathrm{nM}$ (i.e., compound 23). Of the 14 compounds, seven had a similar or lower $K_{I}$ value compared to acetazolamide (AAZ). For hCA XII, five of the tested compounds had a similar or lower $K_{I}$ value compared to acetazolamide. Compound 23 was selective for hCA IX with $\sim$ seven-fold selectivity over hCA XII and at least $\sim 33$-fold selectivity over the off-targets, hCA I and II. Additionally, this compound had a lower $K_{I}$ value compared to acetazolamide (AAZ).

Compound 17 had a cyclohexyl moiety, which was not very well tolerated (Table 1, Figure 1). Adding flexible aliphatic substituents to the cyclohexyl moiety increased the $K_{\mathbf{I}}$ value of the ligand for hCA IX, while adding an aromatic phenyl or bulky tert-butyl substituent (compounds $\mathbf{2 1}$ and 22, respectively) decreased the $\boldsymbol{K}_{\mathbf{I}}$ values (Table 1 , Figure 1 ). A cyclopentyl instead of a cyclohexyl moiety was also not tolerated.

Compound 18, the analog of compound 23 without a cationic nitrogen atom, showed a $\sim 45$-fold higher $K_{\mathrm{I}}$ value compared to compound 23 . This may indicate that a cationic charge was favorable in the ligand binding interactions.

The compounds had, in general, lower $K_{I}$ values for hCA XII, with 18 compounds showing $K_{I}$ smaller than $100 \mathrm{nM}$, including five compounds with $K_{\mathrm{I}}$ smaller than $10 \mathrm{nM}$. Compound 7 had the lowest measured $K_{\mathrm{I}}$ value for hCA XII $\left(K_{\mathrm{I}}: 1.4 \mathrm{nM}\right)$.

The 2-(hydrazinocarbonyl)-3-phenyl-1 $H$-indole-5-sulfonamide was previously tested by members of our group against the tumor-associated hCA IX and XII and the off-targets, hCA I and II [20]. Compared to this previous compound, the newly synthesized hydrazon compounds had higher selectivity for hCA IX/XII compared to the off-targets.

Table 1. Inhibition data of hCA I, II, IX, XII with compounds 4-24 and the standard inhibitor acetazolamide (AAZ) by a stopped flow $\mathrm{CO}_{2}$ hydrase assay.

\begin{tabular}{ccccccc}
\hline & & \multicolumn{4}{c}{$\boldsymbol{K}_{\mathbf{I}}{ }^{*}$ (nM) } \\
\hline Cpd. & $\mathbf{R}$ & $\mathbf{R}_{\mathbf{1}}$ & hCA I & hCA II & hCAIX & hCA XII \\
\hline $\mathbf{4}$ & $\mathrm{H}$ & Thiophene-2-yl & 6667.5 & $>10,000$ & 47.4 & 4.5 \\
$\mathbf{5}$ & $\mathrm{H}$ & 5-Br-thiophene-2-yl & 9408.7 & $>10,000$ & 48.4 & 75.1 \\
$\mathbf{6}$ & $\mathrm{H}$ & 1-CH - -pyrrol-2-yl & 9572 & $>10,000$ & 44.3 & 64.4 \\
$\mathbf{7}$ & $\mathrm{H}$ & pyridine-3-yl & 8592.1 & 8906.7 & 35.8 & 1.4 \\
$\mathbf{8}$ & $\mathrm{H}$ & pyridine-4-yl & 3134.9 & 3487.4 & 15.8 & 6.6 \\
$\mathbf{9}$ & $\mathrm{H}$ & indole-3-yl & $>10,000$ & $>10,000$ & 206.8 & 77.3 \\
$\mathbf{1 0}$ & $\mathrm{H}$ & $\mathrm{H}$ & 7479.2 & 9369.7 & 32.9 & 23.8 \\
$\mathbf{1 1}$ & $\mathrm{H}$ & $\mathrm{CH}_{3}$ & 4056.8 & 5866.8 & 12.9 & 62.6 \\
$\mathbf{1 2}$ & $\mathrm{CH}$ & $\mathrm{CH}_{3}$ & 9558.6 & 8498.4 & 47.9 & 87.9 \\
\hline
\end{tabular}


Table 1. Cont.

\begin{tabular}{ccccccc}
\hline & & \multicolumn{5}{c}{$\boldsymbol{K}_{\mathbf{I}}{ }^{*}(\mathbf{n M})$} \\
\hline $\mathbf{C p d}$. & $\mathbf{R}$ & $\mathbf{R}_{\mathbf{1}}$ & hCA I & hCA II & hCAIX & hCA XII \\
\hline $\mathbf{1 3}$ & $\mathrm{CH}_{3}$ & $\mathrm{C}_{2} \mathrm{H}_{5}$ & 9619.6 & 8029.7 & 42.1 & 92.4 \\
$\mathbf{1 4}$ & $\mathrm{C}_{2} \mathrm{H}_{5}$ & $\mathrm{C}_{2} \mathrm{H}_{5}$ & 9246.3 & 6621.8 & 43.7 & 9.4 \\
$\mathbf{1 5}$ & $\mathrm{CH}_{3}$ & isobuthyl & 8652.3 & 6562.3 & 187.8 & 27.9 \\
$\mathbf{1 6}$ & & cyclopentyl & $>10,000$ & 5960.5 & 476.1 & 97.2 \\
$\mathbf{1 7}$ & & cyclohexyl & $>10,000$ & 5867.9 & 191 & 86.2 \\
$\mathbf{1 8}$ & 4-methylcyclohexyl & $>10,000$ & 8942.5 & 418.9 & 139.1 \\
$\mathbf{1 9}$ & 4-ethylcyclohexyl & $>10,000$ & 1282.7 & 475.3 & 219.7 \\
$\mathbf{2 0}$ & 4-propylcyclohexyl & $>10,000$ & 795.8 & 347.7 & 153.6 \\
$\mathbf{2 1}$ & 4-phenylcyclohexyl & $>10,000$ & 830.7 & 32.3 & 14.8 \\
$\mathbf{2 2}$ & 4-tert-buthylcyclohexyl & $>10,000$ & 3380.8 & 17.4 & 26.6 \\
$\mathbf{2 3}$ & 1-methylpiperidin-4-yl & $>10,000$ & 309 & 9.2 & 62.9 \\
$\mathbf{2 4}$ & 1-benzylpiperidine-4-yl & 7384.7 & 372.4 & 11.7 & 9.4 \\
$\mathbf{A A Z}$ & - & - & 250 & 12.5 & 25 & 5.7 \\
\hline
\end{tabular}

* Mean from three different assays by a stopped flow technique (errors were in the range of 5-10\% of the reported values). $\mathrm{hCA}=$ human carbonic anhydrases, $\mathrm{AAZ}=$ acetazolamide.

A<smiles>[R]C([R])=NNC(=O)c1[nH]c2ccc(S(N)(=O)=O)cc2c1-c1ccccc1</smiles>

B

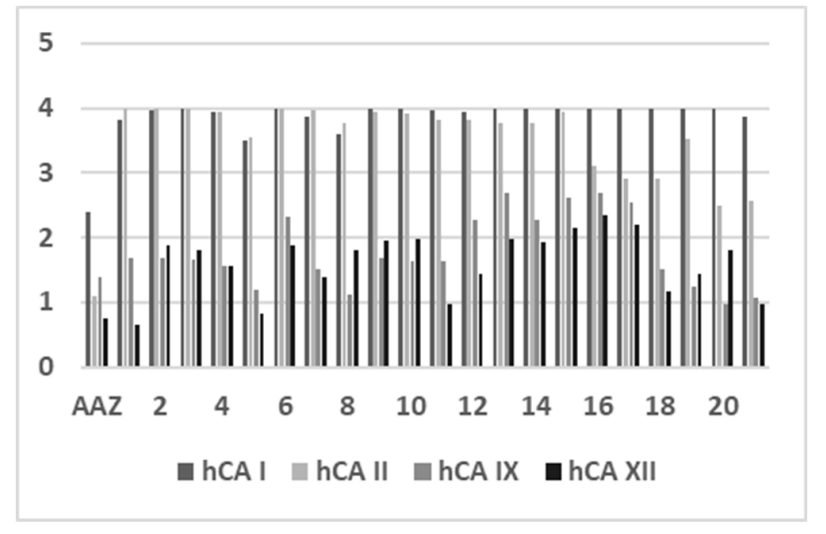

Figure 1. (A) New 2-(hydrazinocarbonyl)-3-phenyl-1H-indole-5-sulfonamide based hydrazone derivatives (substitutions are shown in Table 1). (B) Bar-graph showing the log $K_{\mathrm{I}}$ values of AAZ (acetazolamide) and the tested compounds.

\subsection{Molecular Modeling Studies of hCA IX}

Compound 23 displayed the lowest $K_{\mathrm{I}}$ value for hCA IX of $9.2 \mathrm{nM}$. The sulfonamide moiety formed direct interactions with the $\mathrm{Zn}^{2+}$ ion (tetrahedral orientation) and the backbone of Thr199 (Figure 2; 23 pose in orange). The indole group of the ligand was situated between Gln92 and Leu198. The side chain hydroxyl group of Thr200 pointed with its hydrogen atom towards the centroid of the ligand's phenyl group to interact with the $\pi$-cloud. A hydrogen bond was formed between the ligand's carbonyl group and the side chain of Gln67. The cationic aliphatic ring of the ligand was located between Gln67, Leu91, and Val131. The latter cationic group did not form any interactions with the active site residues. Detailed investigation of the ligand's docked pose and the active site suggested that the ligand cationic aliphatic group could adopt an extended conformation towards Asp132 (Figure 1; 23 pose in purple and Asp132 rotamer in green). The latter residue could adopt another rotameric conformation that reached for the ligand's cationic group, and both a hydrogen bond and an electrostatic interaction could have occurred (distance cationic nitrogen of the ligand and the anionic oxygen atom of Asp132 $2.750 \AA$ ). Such an interaction would be consistent with the enzyme inhibition data for hCA IX in which 23 and 24, compounds with a cationic group, had the lowest $K_{\mathrm{I}}$ values. Compound 18 was an analog of 24 without the cationic nitrogen atom with a much higher $K_{I}$ value. Additional analogs such as 16, 17, and 19-21 similarly had higher $K_{\mathrm{I}}$ values compared to 23. 


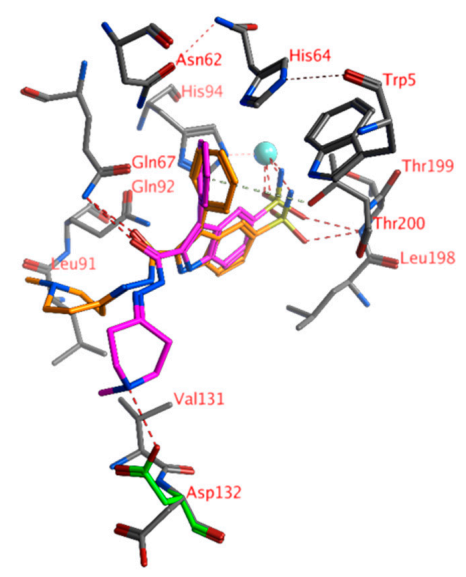

Figure 2. The docked pose of compound 23 (orange and purple) in the active site of hCA IX (3iai). Hydrogen bonds and interactions with the zinc ion are indicated in red dashed lines. H-arene interactions are indicated in yellow dashed lines. The zinc ion is indicated as a turquoise sphere. The Asp132 rotamer that was able to form a hydrogen bond with the ligand is indicated in green sticks.

\subsection{Molecular Modeling Studies of hCA XII}

The lowest $K_{\mathrm{I}}$ value for hCA XII was measured for compound $7\left(K_{\mathrm{I}}: 1.4 \mathrm{nM}\right)$. Again, the sulfonamide moiety formed direct interactions with the $\mathrm{Zn}^{2+}$ ion (tetrahedral orientation) and the backbone of Thr199 (Figure 3). The phenyl and the pyridine groups of the ligands formed $\mathrm{H}$-arene interactions with the hydrogen atoms of Asn62. Further hydrophobic interactions were formed with the side chains of His94, Val121, and Leu198. No hydrogen bonds were formed between the ligand and the active site residues. However, the side chain of Gln92 could adopt a conformation in which a hydrogen bond with the ligand carbonyl group was possible (Figure 3).

In general, the docked poses of the compounds, especially the placement of the sulfonamide and the indole parts of the ligands, were similar due to structural similarity between the compounds and the requirements to form an interaction between the sulfonamide and the zinc ion.

Compounds 18, 19, and 20 had bulky hydrophobic groups that could not form hydrophobic interactions with the active site. This may explain the higher $K_{\mathbf{I}}$ values for these ligands. Remarkably, compound 21 had a lower $K_{I}$ value even though it also had a bulky hydrophobic substituent. The phenyl group of the ligand may have formed hydrophobic interactions with the side chain of Asn69 and a Thr91 rotamer (with the methyl group pointing to the ligand instead of the hydroxyl group; Figure 4). It seems this interaction was not possible for compounds 18, 19, and 20.

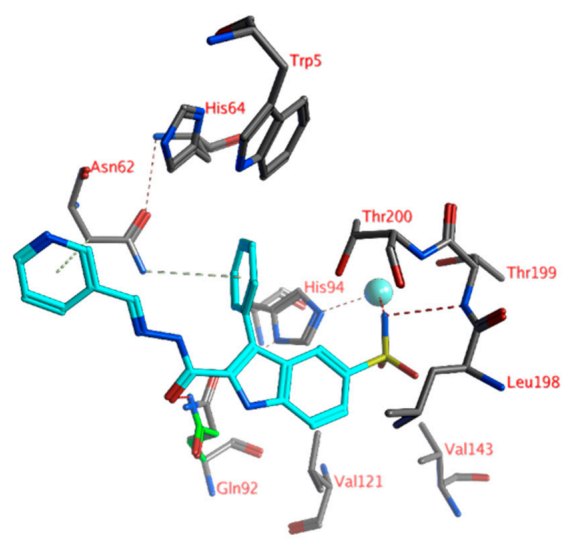

Figure 3. The docked pose of compound 7 (turquoise) in the active site of hCA XII (1jd0). Hydrogen bonds and interactions with the zinc ion are indicated in red dashed lines. H-arene interactions are indicated in yellow dashed lines. The zinc ion is indicated as a turquoise sphere. The Gln92 rotamer that was able to form a hydrogen bond with the ligand is indicated in green sticks. 


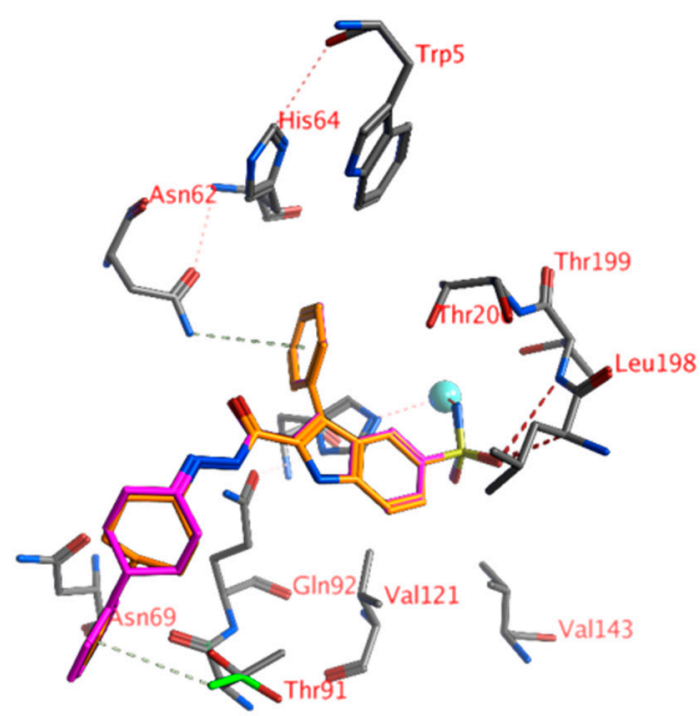

Figure 4. The docked pose of compound 20 (orange) and 21 (purple) in the active site of hCA XII (1jd0). Hydrogen bonds and interactions with the zinc ion are indicated in red dashed lines. $\mathrm{H}$-arene interactions are indicated in yellow dashed lines. The zinc ion is indicated as a turquoise sphere. The Thr91 rotamer that was able to form an $\mathrm{H}$-arene interaction with $\mathbf{2 1}$ is indicated in green sticks.

\subsection{Prediction of ADMET Properties}

The potential mutagenicity [23], the reactivity [24] and Lipinski's parameters (weight, hydrogen bond donor, and acceptor count) of the compounds were calculated using the MOE software package (v2018.0101, Chemical Computing Group Inc., Montreal), and the LogP value [25] was calculated using the ALogPs 2.1 webserver [26]. No violation of Lipinski's rule of five was found [27,28], indicating that the compounds may have passed cell membranes by passive diffusion. Compounds 7, 20, 21, and 23 were not predicted to be mutagenic, but of these four compounds, only compound 7 was not predicted to be reactive. It should be noted, however, that these were theoretical predictions, and experimental validation is still needed.

\section{Materials and Methods}

Melting points were estimated with a Büchi B-540 (BÜCHI Labortechnik AG, Flawil, Switzerland) melting point apparatus in open capillaries and are presented as uncorrected. Elemental analyses were performed on a Thermo Finnigan Flash EA 1112 (Thermo Electron GmbH, Bremen, Germany) elemental analyzer. IR spectra were recorded on KBr discs using a Perkin-Elmer (Waltham, MA, USA) Model 1600 FT-IR spectrometer. ${ }^{1} \mathrm{H}$ NMR, ${ }^{13} \mathrm{C}-\mathrm{NMR}$ (decoupled), and HSQC-2D spectra were obtained on Varian UNITY INOVA 500, Varian Mercury (Agilent, Palo Alto, CA, USA) 400 MHz FT-NMR spectrophotometers using DMSO- $\mathrm{d}_{6}$. The mass spectra were taken on a Waters ZQ micromass LC-MS spectrometer (Waters Corporation, Milford, MA, USA) by using ESI (+) method.

\subsection{Synthetic Procedures}

\subsubsection{2-Benzyl-2-[N-(4-sulfonamidophenyl)hydrazono]ethanoate (1).}

To a solution of 0.01 mole sulfanilamide in $4 \mathrm{~mL}$ of $37 \% \mathrm{HCl}, 10 \mathrm{~mL}$ of $8 \% \mathrm{NaNO}_{2}$ aqueous solution was added dropwise at $0^{\circ} \mathrm{C}$. The solution containing the diazonium salt was poured into an ice-cold mixture of $2.3 \mathrm{~g}$ (a little excess of 0.01 mole) ethyl 2-benzylacetoacetate, $10 \mathrm{~mL}$ of $\mathrm{C}_{2} \mathrm{H}_{5} \mathrm{OH}$, $20 \mathrm{~mL}$ of $\mathrm{H}_{2} \mathrm{O}$, and $2.7 \mathrm{~g}$ of $\mathrm{KOH}$. The mixture was kept cold overnight. The hydrazone, produced as an oil, was separated, dissolved in $\left(\mathrm{C}_{2} \mathrm{H}_{5}\right)_{2} \mathrm{O}$, washed with $\mathrm{H}_{2} \mathrm{O}$, and dried over anhydrous $\mathrm{Na}_{2} \mathrm{SO}_{4}$. $\left(\mathrm{C}_{2} \mathrm{H}_{5}\right)_{2} \mathrm{O}$ was distilled, and the oily residue was treated with $5 \mathrm{~mL}$ of $37 \% \mathrm{HCl}$ and set aside for $5 \mathrm{~h}$ at room temperature. The resulting solid substance was recrystallized from $\mathrm{EtOH}$. 
3.1.2. Ethyl 5-(aminosulfonyl)-3-phenyl-1H-indole-2-carboxylate (2).

A mixture of 0.01 mole ethyl 2-benzyl-2-[N-(4-sulfonamidophenyl)hydrazono]ethanoate and about $10 \mathrm{~mL}$ of $37 \% \mathrm{HCl}$ was heated on a water bath for $4 \mathrm{~h}$, cooled, and poured into $100 \mathrm{~mL}$ of $\mathrm{H}_{2} \mathrm{O}$. The crude product was filtered, washed with $\mathrm{H}_{2} \mathrm{O}$, and recrystallized from $\mathrm{EtOH}$.

\subsubsection{2-(hydrazinylcarbonyl)-3-phenyl-1H-indole-5-sulfonamide (3).}

For this compound, $3.45 \mathrm{~g}$ (0.01 mole) ethyl 5-(aminosulfonyl)-3-phenyl-1 $\mathrm{H}$-indole-2-carboxylate was dissolved in $20 \mathrm{~mL}$ of $\mathrm{C}_{2} \mathrm{H}_{5} \mathrm{OH}$, then $4 \mathrm{~mL}$ of $\mathrm{H}_{2} \mathrm{NNH}_{2}-\mathrm{H}_{2} \mathrm{O}$ was added and refluxed for $6 \mathrm{~h}$, cooled, and kept cold overnight. The resulting crystals were filtered off, washed with $\left(\mathrm{C}_{2} \mathrm{H}_{5}\right)_{2} \mathrm{O}$, and recrystallized from EtOH/DMF.

3.1.4. General procedure for the synthesis of 3-phenyl-5-sulfamoyl-N'-[(heteroaryl)methylidene]$1 H$-indole-2-carbohydrazide/3-phenyl-5-sulfamoyl- $\mathrm{N}^{\prime}$-[(non)substituedcycloalkylidene/alkylidene]$1 H$-indole-2-carbohydrazide derivatives (4-24).

A mixture of 3-phenyl-5-sulfonamidoindole-2-carbohydrazide (3) (0.005 mole), an appropriate aldehyde/heteroaromatic aldehyde/ketone/cyclic ketone ( 0.005 mole), was refluxed in $20 \mathrm{~mL}$ absolute ethanol for 6-12 h. The precipitate obtained was purified either by recrystallization from ethanol or by washing with hot ethanol.

3.1.5. 3-Phenyl-2-\{[2-(thiophene-2-ylmethylidene)hydrazinyl]carbonyl\}-1H-indole-5-sulfonamide (4)

Yellow powder (25\%); mp 309-310 ${ }^{\circ} \mathrm{C}$; IR(KBr): $v$ 3400, 3329, $3250(\mathrm{NH}), 1654(\mathrm{C}=\mathrm{O}) ; 1319,1147$ $(\mathrm{S}=\mathrm{O}) ;{ }^{1} \mathrm{H}$ NMR $\left(\mathrm{DMSO}_{\mathrm{d}} / 500 \mathrm{MHz} \delta(\mathrm{ppm}): 7.13\left(1 \mathrm{H}, \mathrm{s}\right.\right.$, thiophene $\left.\mathrm{C}_{4}-\mathrm{H}\right), 7.20\left(2 \mathrm{H}, \mathrm{s}, \mathrm{SO}_{2} \mathrm{NH}_{2}\right), 7.39$ $\left(1 \mathrm{H}\right.$, br s, thiophene $\left.\mathrm{C}_{3}-\mathrm{H}\right), 7.44\left(1 \mathrm{H}, \mathrm{br} \mathrm{s}\right.$, thiophene $\left.\mathrm{C}_{5}-\mathrm{H}\right), 7.50-7.57(5 \mathrm{H}, \mathrm{m}, 3-$ phenyl C-H), $7.64(1 \mathrm{H}$, $\mathrm{d}, \mathrm{J}=8.79 \mathrm{~Hz}$, indole $\left.\mathrm{C}_{7}-\mathrm{H}\right), 7.74\left(1 \mathrm{H}, \mathrm{d}, \mathrm{J}=8.79 \mathrm{~Hz}\right.$, indole $\left.\mathrm{C}_{6}-\mathrm{H}\right), 8.14\left(1 \mathrm{H}, \mathrm{s}\right.$, indole $\left.\mathrm{C}_{4}-\mathrm{H}\right), 8.31(1 \mathrm{H}, \mathrm{s}$, $\mathrm{N}=\mathrm{CH}), 11.44(1 \mathrm{H}, \mathrm{s}, \mathrm{CONH}), 12.35\left(1 \mathrm{H}, \mathrm{s}\right.$, indole NH). ${ }^{13} \mathrm{C}-\mathrm{NMR}$ (HSQC-2D, DMSO-d $\left.\mathrm{d}_{6}, 125 \mathrm{MHz}\right)$ $\delta(\mathrm{ppm}): 113.19$ [ind. $\mathrm{C}_{7}-7.64\left(1 \mathrm{H}, \mathrm{d}, \mathrm{J}=8.79 \mathrm{~Hz}\right.$, indole $\left.\mathrm{C}_{7}-\mathrm{H}\right)$ ], 119.06 [ind. $\mathrm{C}_{4}-8.14(1 \mathrm{H}, \mathrm{s}$, indole $\left.\mathrm{C}_{4}-\mathrm{H}\right)$ ], 121.79 [ind. $\mathrm{C}_{6}-7.74\left(1 \mathrm{H}, \mathrm{d}, \mathrm{J}=8.79 \mathrm{~Hz}\right.$, indole $\left.\mathrm{C}_{6}-\mathrm{H}\right)$ ], 127.59 [thiophene $\mathrm{C}_{3}-7.39(1 \mathrm{H}$, br s, thiophene $\left.\mathrm{C}_{3}-\mathrm{H}\right)$ ], 128.32 [thiophene $\mathrm{C}_{4}-7.13\left(1 \mathrm{H}\right.$, s, thiophene $\left.\mathrm{C}_{4}-\mathrm{H}\right)$ ], 129.05, 129.77, 130,16 [3-Phenyl C - 7.50-7.57 (5H, m, 3-phenyl C-H)], 131.88 [thiophene $\mathrm{C}_{5}-7.44\left(1 \mathrm{H}\right.$, br s, thiophene $\left.\mathrm{C}_{5}-\mathrm{H}\right)$ ], 143.19 $[\mathrm{N}=\mathrm{CH}-8.31(1 \mathrm{H}, \mathrm{s}, \mathrm{N}=\mathrm{CH})]$. Anal. Calcd. for $\mathrm{C}_{20} \mathrm{H}_{16} \mathrm{~N}_{4} \mathrm{O}_{3} \mathrm{~S}_{2}$ (424.496): $\mathrm{C}, 56.59 ; \mathrm{H}, 3.80 ; \mathrm{N}, 13.20 ; \mathrm{S}$, 15.11. Found: $\mathrm{C}, 56.27 ; \mathrm{H}, 3.60 ; \mathrm{N}, 11.61 ; \mathrm{S}, 15.43$.

3.1.6. 2-(\{2-[(5-Bromothiophene-2-yl)methylidene]hydrazinyl $\}$ carbonyl)-3-phenyl-1H-indole-5-sulfonamide (5)

Yellow powder (30\%); mp 272-273 ${ }^{\circ} \mathrm{C}$; $\mathrm{IR}(\mathrm{KBr}): \mathrm{v} 3423,3300,3217(\mathrm{NH}), 1654(\mathrm{C}=\mathrm{O}) ; 1334,1153$ $(\mathrm{S}=\mathrm{O}) ;{ }^{1} \mathrm{H}$ NMR $\left(\mathrm{DMSO}_{6} / 500 \mathrm{MHz} \delta(\mathrm{ppm}): 7.20\left(2 \mathrm{H}, \mathrm{s}, \mathrm{SO}_{2} \mathrm{NH}_{2}\right), 7.26\left(1 \mathrm{H}, \mathrm{br} \mathrm{s}\right.\right.$, thiophene $\left.\mathrm{C}_{4}-\mathrm{H}\right)$, $7.31\left(1 \mathrm{H}\right.$, br s, thiophene $\left.\mathrm{C}_{3}-\mathrm{H}\right), 7.40(1 \mathrm{H}$, br s, 3-phenyl C-H), $7.52(4 \mathrm{H}$, br s, 3-phenyl C-H), $7.64(1 \mathrm{H}, \mathrm{d}$, $\mathrm{J}=8.78 \mathrm{~Hz}$, indole $\left.\mathrm{C}_{7}-\mathrm{H}\right), 7.73\left(1 \mathrm{H}, \mathrm{dd}, \mathrm{J}=9.04,1.46 \mathrm{~Hz}\right.$, indole $\left.\mathrm{C}_{6}-\mathrm{H}\right), 8.13\left(1 \mathrm{H}\right.$, s, indole $\left.\mathrm{C}_{4}-\mathrm{H}\right), 8.24(1 \mathrm{H}$, $\mathrm{s}, \mathrm{N}=\mathrm{CH}), 11.52(1 \mathrm{H}, \mathrm{s}, \mathrm{CONH}), 12.32(1 \mathrm{H}$, s, indole $\mathrm{NH})$. Anal. Calcd. for $\mathrm{C}_{20} \mathrm{H}_{15} \mathrm{BrN}_{4} \mathrm{O}_{3} \mathrm{~S}_{2}(503.392)$ : C, 47.72; H, 3.00; N, 11.13; S, 12.74. Found: C, 47.82; H, 3.30; N, 11.41; S, 12.90.

\subsubsection{2-(\{2-[(1-Methyl-1H-pyrrol-2-yl)methylidene]hydrazinyl\}carbonyl)-3-phenyl-1H-indole-5-sulfonamide (6)}

Yellow powder (40\%); mp 298-299 다 $\mathrm{IR}(\mathrm{KBr}): v$ 3419, 3313, 3253, $3213(\mathrm{NH}), 1645(\mathrm{C}=\mathrm{O}) ; 1307$, $1157(\mathrm{~S}=\mathrm{O}) ;{ }^{1} \mathrm{H}$ NMR (DMSO-d $6 / 500 \mathrm{MHz} \delta(\mathrm{ppm}): 3.84\left(3 \mathrm{H}, \mathrm{s}\right.$, pyrrol 1- $\left.\mathrm{CH}_{3}\right), 6.10(1 \mathrm{H}, \mathrm{s}$, pyrrol $\left.\mathrm{C}_{3}-\mathrm{H}\right), 6.48\left(1 \mathrm{H}, \mathrm{s}\right.$, pyrrol $\left.\mathrm{C}_{4}-\mathrm{H}\right), 6.98\left(1 \mathrm{H}, \mathrm{s}\right.$, pyrrol $\left.\mathrm{C}_{5}-\mathrm{H}\right), 7.20\left(2 \mathrm{H}, \mathrm{s}, \mathrm{SO}_{2} \mathrm{NH}_{2}\right), 7.41(2 \mathrm{H}, \mathrm{d}, \mathrm{J}=6.83$ $\mathrm{Hz}$, 3-phenyl C-H), 7.51-7.56 (3H, m, 3-phenyl C-H), $7.64\left(1 \mathrm{H}, \mathrm{d}, \mathrm{J}=8.78 \mathrm{~Hz}\right.$, indole $\left.\mathrm{C}_{7}-\mathrm{H}\right), 7.74(1 \mathrm{H}, \mathrm{d}$, $\mathrm{J}=8.30 \mathrm{~Hz}$, indole $\left.\mathrm{C}_{6}-\mathrm{H}\right), 8.10\left(1 \mathrm{H}\right.$, s, indole $\left.\mathrm{C}_{4}-\mathrm{H}\right), 8.13(1 \mathrm{H}, \mathrm{s}, \mathrm{N}=\mathrm{CH}), 11.13(1 \mathrm{H}, \mathrm{s}, \mathrm{CONH}), 12.31(1 \mathrm{H}$, $\mathrm{s}$, indole $\mathrm{NH}$ ). Anal. Calcd. for $\mathrm{C}_{21} \mathrm{H}_{19} \mathrm{~N}_{5} \mathrm{O}_{3} \mathrm{~S}$ (421.472): $\mathrm{C}, 59.84 ; \mathrm{H}, 4.54 ; \mathrm{N}, 16.62 ; \mathrm{S}, 7.61$. Found: $\mathrm{C}$, $59.67 ; \mathrm{H}, 4.62 ; \mathrm{N}, 16.81 ; \mathrm{S}, 7.83$. 
3.1.8. 3-Phenyl-2-\{[2-(pyridine-3-ylmethylidene)hydrazinyl]carbonyl\}-1H-indole-5-sulfonamide (7)

Ivory powder (60\%); $\mathrm{mp} 287.5-287.8^{\circ} \mathrm{C} ; \mathrm{IR}(\mathrm{KBr}): v$ 3360, 3275, $3190(\mathrm{NH}), 1643(\mathrm{C}=\mathrm{O}) ; 1340,1159$ $(\mathrm{S}=\mathrm{O}) ;{ }^{1} \mathrm{H}$ NMR $\left(\mathrm{DMSO}_{6} / 500 \mathrm{MHz} \delta(\mathrm{ppm}): 7.19\left(2 \mathrm{H}, \mathrm{s}, \mathrm{SO}_{2} \mathrm{NH}_{2}\right), 7.32-7.53(6 \mathrm{H}, \mathrm{m}, 3\right.$-phenyl C-H and pyridine $\left.\mathrm{C}_{5}-\mathrm{H}\right), 7.65\left(1 \mathrm{H}, \mathrm{d}, \mathrm{J}=8.78 \mathrm{~Hz}\right.$, indole $\left.\mathrm{C}_{7}-\mathrm{H}\right), 7.74\left(1 \mathrm{H}, \mathrm{d}, \mathrm{J}=8.30 \mathrm{~Hz}\right.$, indole $\left.\mathrm{C}_{6}-\mathrm{H}\right), 8.14$ $\left(2 \mathrm{H}\right.$, br s, indole $\mathrm{C}_{4}-\mathrm{H}$ and $\left.\mathrm{N}=\mathrm{CH}\right), 8.48\left(1 \mathrm{H}, \mathrm{br} \mathrm{s}\right.$, pyridine $\left.\mathrm{C}_{4}-\mathrm{H}\right), 8.60\left(1 \mathrm{H}, \mathrm{s}\right.$, pyridine $\left.\mathrm{C}_{6}-\mathrm{H}\right), 8.82$ $\left(1 \mathrm{H}, \mathrm{s}\right.$, pyridine $\left.\mathrm{C}_{2}-\mathrm{H}\right), 11.64(1 \mathrm{H}, \mathrm{s}, \mathrm{CONH}), 12.38(1 \mathrm{H}$, s, indole $\mathrm{NH})$. Anal. Calcd. for $\mathrm{C}_{21} \mathrm{H}_{17} \mathrm{~N}_{5} \mathrm{O}_{3} \mathrm{~S}$ (419.456): C, 60.13; H, 4.09; N, 16.70; S, 7.64. Found: C, 59.80; H, 3.93; N, 16.61; S, 7.43.

\subsubsection{3-Phenyl-2-\{[2-(pyridine-4-ylmethylidene)hydrazinyl]carbonyl\}-1H-indole-5-sulfonamide (8)}

Yellow powder (40\%); mp 271.5-271.7 ${ }^{\circ} \mathrm{C}$; $\mathrm{IR}(\mathrm{KBr}): v 3236,3178,3163(\mathrm{NH}), 1666(\mathrm{C}=\mathrm{O})$; 1321, 1151 (S=O); ${ }^{1} \mathrm{H}$ NMR (DMSO-d $6 / 500 \mathrm{MHz} \delta$ (ppm): $7.21\left(2 \mathrm{H}, \mathrm{s}, \mathrm{SO}_{2} \mathrm{NH}_{2}\right)$, 7.29-7.58 (7H, m, 3-phenyl $\mathrm{C}-\mathrm{H}$ and pyridine $\left.\mathrm{C}_{3,5}-\mathrm{H}\right), 7.65\left(1 \mathrm{H}, \mathrm{d}, \mathrm{J}=8.30 \mathrm{~Hz}\right.$, indole $\left.\mathrm{C}_{7}-\mathrm{H}\right), 7.75(1 \mathrm{H}, \mathrm{dd}, \mathrm{J}=8.78,1.47 \mathrm{~Hz}$, indole $\left.\mathrm{C}_{6}-\mathrm{H}\right), 8.09\left(1 \mathrm{H}, \mathrm{s}\right.$, indole $\left.\mathrm{C}_{4}-\mathrm{H}\right), 8.15(1 \mathrm{H}, \mathrm{br} \mathrm{s}, \mathrm{N}=\mathrm{CH}), 8.62\left(2 \mathrm{H}\right.$, br s, pyridine $\left.\mathrm{C}_{2,6}-\mathrm{H}\right), 11.75(1 \mathrm{H}, \mathrm{s}$, $\mathrm{CONH}), 12.38(1 \mathrm{H}$, s, indole $\mathrm{NH})$. Anal. Calcd. for $\mathrm{C}_{21} \mathrm{H}_{17} \mathrm{~N}_{5} \mathrm{O}_{3} \mathrm{~S}$ (419.456): $\mathrm{C}, 60.13 ; \mathrm{H}, 4.09 ; \mathrm{N}, 16.70$; S, 7.64. Found: C, 60.33; H, 4.35; N, 16.91; S, 7.93.

\subsubsection{3-Phenyl-2-\{[2-(1H-indole-3-ylmethylidene)hydrazinyl]carbonyl\}-1H-indole-5-sulfonamide (9)}

Yellow crystal (20\%); mp 264-265 ${ }^{\circ} \mathrm{C}$; IR(KBr): v 3373, 3313, $3211(\mathrm{NH}), 1643(\mathrm{C}=\mathrm{O}) ; 1319,1151$ $(\mathrm{S}=\mathrm{O}) ;{ }^{1} \mathrm{H}$ NMR (DMSO- $\mathrm{d}_{6} / 500 \mathrm{MHz} \delta(\mathrm{ppm}): 7.15-7.17\left(4 \mathrm{H}, \mathrm{m}\right.$, 2.indole $\mathrm{C}_{5,6}-\mathrm{H}$ and $\left.\mathrm{SO}_{2} \mathrm{NH}_{2}\right), 7.30-7.45$ $\left(2 \mathrm{H}, \mathrm{m}, 3\right.$-phenyl $\mathrm{C}_{4}-\mathrm{H}$ and 2.indole $\left.\mathrm{C}_{7}-\mathrm{H}\right), 7.51\left(2 \mathrm{H}, \mathrm{t}, \mathrm{J}=7.81 \mathrm{~Hz}, 3\right.$-phenyl $\left.\mathrm{C}_{3,5}-\mathrm{H}\right), 7.58(2 \mathrm{H}, \mathrm{d}, \mathrm{J}=7.32$ $\mathrm{Hz}$, 3-phenyl $\left.\mathrm{C}_{2,6}-\mathrm{H}\right), 7.64\left(1 \mathrm{H}, \mathrm{d}, \mathrm{J}=8.78 \mathrm{~Hz}\right.$, indole $\left.\mathrm{C}_{7}-\mathrm{H}\right), 7.70\left(1 \mathrm{H}, \mathrm{dd}, \mathrm{J}=8.78,1.46 \mathrm{~Hz}\right.$, indole $\left.\mathrm{C}_{6}-\mathrm{H}\right)$, $7.79\left(1 \mathrm{H}, \mathrm{d}, \mathrm{J}=2.93 \mathrm{~Hz}\right.$, 2.indole $\left.\mathrm{C}_{2}-\mathrm{H}\right), 8.13\left(1 \mathrm{H}\right.$, s, indole $\left.\mathrm{C}_{4}-\mathrm{H}\right), 8.25(1 \mathrm{H}, \mathrm{s}, \mathrm{N}=\mathrm{CH}), 8.26(1 \mathrm{H}, \mathrm{d}, \mathrm{J}=7.81$ $\mathrm{Hz}$, 2. indole $\left.\mathrm{C}_{4}-\mathrm{H}\right), 11.07(1 \mathrm{H}, \mathrm{s}, \mathrm{CONH}), 11.59(1 \mathrm{H}, \mathrm{s}, 2$. indole $\mathrm{NH}), 12.34(1 \mathrm{H}, \mathrm{s}$, indole $\mathrm{NH})$. Anal. Calcd. for $\mathrm{C}_{24} \mathrm{H}_{19} \mathrm{~N}_{5} \mathrm{O}_{3} \mathrm{~S}$ (457.504): $\mathrm{C}, 63.01 ; \mathrm{H}, 4.19 ; \mathrm{N}, 15.31 ; \mathrm{S}, 7.01$. Found: $\mathrm{C}, 59.87 ; \mathrm{H}, 4.50 ; \mathrm{N}, 15.61$; S, 7.33 .

\subsubsection{2-[(2-Methylidenehydrazinyl)carbonyl]-3-phenyl-1H-indole-5-sulfonamide (10)}

White powder (30\%); mp 248.5-248.8 ${ }^{\circ} \mathrm{C}$; IR(KBr): v $3244(\mathrm{NH}), 1645(\mathrm{C}=\mathrm{O}) ; 1323,1149(\mathrm{~S}=\mathrm{O}) ;{ }^{1} \mathrm{H}$ NMR (DMSO-d $\mathrm{d}_{6} / 500 \mathrm{MHz} \delta(\mathrm{ppm}): 7.17\left(2 \mathrm{H}, \mathrm{s}, \mathrm{SO}_{2} \mathrm{NH}_{2}\right), 7.35-7.49(5 \mathrm{H}, \mathrm{m}, 3-$ phenyl C-H), $7.62(1 \mathrm{H}, \mathrm{d}$, $\mathrm{J}=8.21 \mathrm{~Hz}$, indole $\left.\mathrm{C}_{7}-\mathrm{H}\right), 7.70\left(1 \mathrm{H}, \mathrm{d}, \mathrm{J}=8.21 \mathrm{~Hz}\right.$, indole $\left.\mathrm{C}_{6}-\mathrm{H}\right), 8.10\left(1 \mathrm{H}\right.$, s, indole $\left.\mathrm{C}_{4}-\mathrm{H}\right), 8.21,8.24(2 \mathrm{H}$, $\left.2 \mathrm{~d}, \mathrm{~J}=3.52 \mathrm{~Hz}, \mathrm{~N}=\mathrm{CH}_{2}\right), 11.26(1 \mathrm{H}, \mathrm{s}, \mathrm{CONH}), 12.14(1 \mathrm{H}$, s, indole $\mathrm{NH})$. Anal. Calcd. for $\mathrm{C}_{16} \mathrm{H}_{14} \mathrm{~N}_{4} \mathrm{O}_{3} \mathrm{~S}$ (342.372): C, 56.13; H, 4.12; N, 16.36; S, 9.37. Found: C, 56.50; H, 3.93; N, 16.60; S, 9.67.

\subsubsection{2-[(2-Ethylidenehydrazinyl)carbonyl]-3-phenyl-1H-indole-5-sulfonamide (11)}

Ivory powder (27\%); mp 250-250.1 ${ }^{\circ} \mathrm{C}$; IR(KBr): v 3302, $3221(\mathrm{NH}), 1670(\mathrm{C}=\mathrm{O}) ; 1323,1145(\mathrm{~S}=\mathrm{O})$; ${ }^{1} \mathrm{H}$ NMR (DMSO-d $\mathrm{d}_{6} / 500 \mathrm{MHz} \delta(\mathrm{ppm}): 1.88\left(3 \mathrm{H}, \mathrm{d}, \mathrm{J}=3.90 \mathrm{~Hz},-\mathrm{CH}_{3}\right), 7.18\left(2 \mathrm{H}, \mathrm{s}, \mathrm{SO}_{2} \mathrm{NH}_{2}\right), 7.34-7.44$ $\left(2 \mathrm{H}, \mathrm{m}, 3\right.$-phenyl $\mathrm{C}_{4}-\mathrm{H}$ and $\left.\mathrm{N}=\mathrm{CH}\right), 7.46-7.54\left(4 \mathrm{H}, \mathrm{m}, 3\right.$-phenyl $\left.\mathrm{C}_{2,3,5,6}-\mathrm{H}\right), 7.61(1 \mathrm{H}, \mathrm{d}, \mathrm{J}=8.30 \mathrm{~Hz}$, indole $\left.\mathrm{C}_{7}-\mathrm{H}\right), 7.75\left(1 \mathrm{H}, \mathrm{d}, \mathrm{J}=8.78 \mathrm{~Hz}\right.$, indole $\left.\mathrm{C}_{6}-\mathrm{H}\right), 8.10\left(1 \mathrm{H}, \mathrm{d}, \mathrm{J}=1.95 \mathrm{~Hz}\right.$, indole $\left.\mathrm{C}_{4}-\mathrm{H}\right), 11.00(1 \mathrm{H}, \mathrm{s}, \mathrm{CONH})$, $12.31(1 \mathrm{H}, \mathrm{s}$, indole $\mathrm{NH}) .{ }^{13} \mathrm{CNMR}$ (proton decoupled, DMSO-d $\left.\mathrm{d}_{6}, 150 \mathrm{MHz}\right) \delta(\mathrm{ppm}): 56.15\left(-\mathrm{CH}_{3}\right)$, 112.69 (ind. $C_{7}$ ), 118.64 (ind. $C_{4}$ ), 119.20 (ind. $C_{3}$ ), 121.26 (ind. $C_{6}$ ), 125.46 (ind. $C_{3 a}$ ), 127.17 (3-Phenyl $\mathrm{C}_{4}$ ), 128.65 (3-Phenyl $\mathrm{C}_{3 / 5}$ ), 129.19 (ind. $\mathrm{C}_{2}$ ), 129.64 (3-Phenyl $\mathrm{C}_{2 / 6}$ ), 133.03 (ind. $\mathrm{C}_{5}$ ), 136.74 (3-Phenyl $\left.\mathrm{C}_{1}\right), 139.05$ (ind. $\left.\mathrm{C}_{7 \mathrm{a}}\right), 148.67(\mathrm{~N}=\mathrm{C}), 157.75(\mathrm{CONH})$. Anal. Calcd. for $\mathrm{C}_{17} \mathrm{H}_{16} \mathrm{~N}_{4} \mathrm{O}_{3} \mathrm{~S}$ (356.398): $\mathrm{C}, 57.29$; $\mathrm{H}, 4.52 ; \mathrm{N}, 15.72 ; \mathrm{S}, 9.00$. Found: C, 56.96; H, 4.13; N, 16.05; S, 9.37. m/z ESI (positive) $357.36(\mathrm{M}+\mathrm{H})^{+}$.

\subsubsection{3-Phenyl-2-\{[2-(propan-2-ylidene)hydrazinyl]carbonyl\}-1H-indole-5-sulfonamide (12)}

White powder (30\%); mp 287.9-288 ${ }^{\circ} \mathrm{C}$; $\mathrm{IR}(\mathrm{KBr}): v$ 3334, 3305, $3217(\mathrm{NH}), 1668(\mathrm{C}=\mathrm{O}) ; 1325$, $1147(\mathrm{~S}=\mathrm{O}) ;{ }^{1} \mathrm{H}$ NMR $\left(\mathrm{DMSO}_{-} \mathrm{d}_{6} / 500 \mathrm{MHz} \delta(\mathrm{ppm}): 1.27\left(3 \mathrm{H}, \mathrm{s},-\mathrm{CH}_{3}\right), 1.89\left(3 \mathrm{H}, \mathrm{s},-\mathrm{CH}_{3}\right), 7.17(2 \mathrm{H}, \mathrm{s}\right.$, $\left.\mathrm{SO}_{2} \mathrm{NH}_{2}\right), 7.45-7.56\left(5 \mathrm{H}, \mathrm{m}, 3\right.$-phenyl C-H), $7.62\left(1 \mathrm{H}, \mathrm{d}, \mathrm{J}=8.30 \mathrm{~Hz}\right.$, indole $\left.\mathrm{C}_{7}-\mathrm{H}\right), 7.72(1 \mathrm{H}, \mathrm{dd}, \mathrm{J}=8.30$, $1.95 \mathrm{~Hz}$, indole $\left.\mathrm{C}_{6}-\mathrm{H}\right), 7.91\left(1 \mathrm{H}\right.$, s, indole $\left.\mathrm{C}_{4}-\mathrm{H}\right), 9.24(1 \mathrm{H}, \mathrm{s}, \mathrm{CONH}), 12.39(1 \mathrm{H}$, s, indole $\mathrm{NH})$. Anal. 
Calcd. for $\mathrm{C}_{18} \mathrm{H}_{18} \mathrm{~N}_{4} \mathrm{O}_{3} \mathrm{~S}$ (370.425): C, 58.36; H, 4.90; N, 15.12; S, 8.66. Found: C, 58.76; H, 5.13; N, 15.05; S, 9.01 .

\subsubsection{2-\{[2-(Butan-2-ylidene)hydrazinyl]carbonyl\}-3-phenyl-1H-indole-5-sulfonamide (13)}

White powder (78\%); mp 285.5-285.6 ${ }^{\circ} \mathrm{C}$; $\mathrm{IR}(\mathrm{KBr}): v 3360,3304,3224(\mathrm{NH}), 1680(\mathrm{C}=\mathrm{O}) ; 1315,1151$ $(\mathrm{S}=\mathrm{O}) ;{ }^{1} \mathrm{H}$ NMR $\left(\mathrm{DMSO}_{-} \mathrm{d}_{6} / 500 \mathrm{MHz} \delta(\mathrm{ppm}): 0.93-1.04\left(3 \mathrm{H}, \mathrm{m},-\mathrm{CH}_{3}\right), 1.26\left(3 \mathrm{H}, \mathrm{s},-\mathrm{CH}_{3}\right), 2.20(2 \mathrm{H}\right.$, $\left.\mathrm{d}, \mathrm{J}=4.88 \mathrm{~Hz},-\mathrm{CH}_{2}-\right), 7.17\left(2 \mathrm{H}, \mathrm{s}, \mathrm{SO}_{2} \mathrm{NH}_{2}\right), 7.45-7.57(5 \mathrm{H}, \mathrm{m}, 3$-phenyl C-H), $7.63(1 \mathrm{H}, \mathrm{d}, \mathrm{J}=8.79 \mathrm{~Hz}$, indole $\left.\mathrm{C}_{7}-\mathrm{H}\right), 7.71\left(1 \mathrm{H}, \mathrm{dd}, \mathrm{J}=8.79,1.46 \mathrm{~Hz}\right.$, indole $\left.\mathrm{C}_{6}-\mathrm{H}\right), 7.91\left(1 \mathrm{H}\right.$, s, indole $\left.\mathrm{C}_{4}-\mathrm{H}\right), 9.19(1 \mathrm{H}, \mathrm{s}, \mathrm{CONH})$, $12.35(1 \mathrm{H}$, s, indole $\mathrm{NH})$. Anal. Calcd. for $\mathrm{C}_{19} \mathrm{H}_{20} \mathrm{~N}_{4} \mathrm{O}_{3} \mathrm{~S}$ (384.452): $\mathrm{C}, 59.36 ; \mathrm{H}, 5.24 ; \mathrm{N}, 14.57 ; \mathrm{S}, 8.34$. Found: C, 59.56; H, 5.36; N, 14.15; S, 8.71.

\subsubsection{2-\{[2-(Pentan-3-ylidene)hydrazinyl]carbonyl\}-3-phenyl-1H-indole-5-sulfonamide (14)}

White powder (60\%); mp 288.2-288.3 ${ }^{\circ} \mathrm{C}$; $\mathrm{IR}(\mathrm{KBr}): v$ 3356, 3300, $3221(\mathrm{NH}), 1680(\mathrm{C}=\mathrm{O}) ; 1319,1151$ $(\mathrm{S}=\mathrm{O}) ;{ }^{1} \mathrm{H}$ NMR $\left(\mathrm{DMSO}_{6} / 500 \mathrm{MHz} \delta(\mathrm{ppm}): 0.63\left(3 \mathrm{H}, \mathrm{s},-\mathrm{CH}_{3}\right), 1.00\left(3 \mathrm{H}, \mathrm{s},-\mathrm{CH}_{3}\right), 1.60(2 \mathrm{H}, \mathrm{d}, \mathrm{J}=6.04\right.$ $\left.\mathrm{Hz},-\mathrm{CH}_{2}-\right), 2.20\left(2 \mathrm{H}, \mathrm{d}, \mathrm{J}=6.04 \mathrm{~Hz},-\mathrm{CH}_{2}-\right), 7.15\left(2 \mathrm{H}, \mathrm{s}, \mathrm{SO}_{2} \mathrm{NH}_{2}\right), 7.46-7.58(5 \mathrm{H}, \mathrm{m}, 3-$ phenyl C-H), 7.62 $\left(1 \mathrm{H}, \mathrm{d}, \mathrm{J}=8.78 \mathrm{~Hz}\right.$, indole $\left.\mathrm{C}_{7}-\mathrm{H}\right), 7.71\left(1 \mathrm{H}, \mathrm{dd}, \mathrm{J}=8.23,1.65 \mathrm{~Hz}\right.$, indole $\left.\mathrm{C}_{6}-\mathrm{H}\right), 7.85\left(1 \mathrm{H}\right.$, s, indole $\left.\mathrm{C}_{4}-\mathrm{H}\right)$, $9.20(1 \mathrm{H}, \mathrm{s}, \mathrm{CONH}), 12.39(1 \mathrm{H}, \mathrm{s}$, indole $\mathrm{NH})$. Anal. Calcd. for $\mathrm{C}_{20} \mathrm{H}_{22} \mathrm{~N}_{4} \mathrm{O}_{3} \mathrm{~S}$ (398.478): C, 60.28; $\mathrm{H}$, 5.56; N, 14.06; S, 8.05. Found: C, 59.96; H, 5.16; N, 14.40; S, 8.36.

3.1.16. 2-\{[2-(4-Methylpentan-2-ylidene)hydrazinyl]carbonyl\}-3-phenyl-1H-indole-5-sulfonamide (15)

White powder (50\%); mp 255.1-255.2 ${ }^{\circ} \mathrm{C}$; IR(KBr): v 3352, 3321, $3265(\mathrm{NH}), 1674(\mathrm{C}=\mathrm{O}) ; 1332$, $1151(\mathrm{~S}=\mathrm{O}) ;{ }^{1} \mathrm{H}$ NMR (DMSO-d $6 / 500 \mathrm{MHz} \delta(\mathrm{ppm}): 0.83\left(6 \mathrm{H}, \mathrm{d}, \mathrm{J}=5.49 \mathrm{~Hz},-\mathrm{CH}\left(\mathrm{CH}_{3}\right)_{2}\right), 1.24(3 \mathrm{H}, \mathrm{s}$, $\left.-\mathrm{CH}_{3}\right), 1.84-1.88(1 \mathrm{H}, \mathrm{m},-\mathrm{CH}-), 2.05\left(2 \mathrm{H}, \mathrm{d}, \mathrm{J}=5.49 \mathrm{~Hz},-\mathrm{CH}_{2}-\right), 7.16\left(2 \mathrm{H}, \mathrm{s}, \mathrm{SO}_{2} \mathrm{NH}_{2}\right), 7.47-7.56(5 \mathrm{H}, \mathrm{m}$, 3-phenyl C-H), $7.62\left(1 \mathrm{H}, \mathrm{d}, \mathrm{J}=8.78 \mathrm{~Hz}\right.$, indole $\left.\mathrm{C}_{7}-\mathrm{H}\right), 7.72\left(1 \mathrm{H}, \mathrm{dd}, \mathrm{J}=8.78,1.65 \mathrm{~Hz}\right.$, indole $\left.\mathrm{C}_{6}-\mathrm{H}\right), 7.91$ $\left(1 \mathrm{H}\right.$, s, indole $\left.\mathrm{C}_{4}-\mathrm{H}\right), 9.20(1 \mathrm{H}, \mathrm{s}, \mathrm{CONH}), 12.36(1 \mathrm{H}$, s, indole $\mathrm{NH})$. Anal. Calcd. for $\mathrm{C}_{21} \mathrm{H}_{24} \mathrm{~N}_{4} \mathrm{O}_{3} \mathrm{~S}$ (412.505): C, 61.14; H, 5.86; N, 13.58; S, 7.77. Found: C, 60.83; H, 5.50; N, 13.35; S, 7.56.

\subsubsection{2-[(2-Cyclopentylidenehydrazinyl)carbonyl]-3-phenyl-1H-indole-5-sulfonamide (16)}

White powder (84\%); $\mathrm{mp} 313.1-313.2{ }^{\circ} \mathrm{C}$; $\mathrm{IR}(\mathrm{KBr}): v 3336,3234,3138(\mathrm{NH}), 1658(\mathrm{C}=\mathrm{O})$; 1344, $1153(\mathrm{~S}=\mathrm{O}) ;{ }^{1} \mathrm{H}$ NMR (DMSO-d $6 / 500 \mathrm{MHz} \delta(\mathrm{ppm}): 1.50-1.65(6 \mathrm{H}, \mathrm{m},-$ cyclopentyl C-H), $2.30(2 \mathrm{H}$, $\mathrm{m}$, -cyclopentyl C-H), $7.17\left(2 \mathrm{H}, \mathrm{s}, \mathrm{SO}_{2} \mathrm{NH}_{2}\right), 7.50-7.59(5 \mathrm{H}, \mathrm{m}, 3$-phenyl C-H), $7.61(1 \mathrm{H}, \mathrm{d}, \mathrm{J}=8.78 \mathrm{~Hz}$, indole $\left.\mathrm{C}_{7}-\mathrm{H}\right), 7.71\left(1 \mathrm{H}, \mathrm{dd}, \mathrm{J}=8.78,0.97 \mathrm{~Hz}\right.$, indole $\left.\mathrm{C}_{6}-\mathrm{H}\right), 7.89\left(1 \mathrm{H}\right.$, s, indole $\left.\mathrm{C}_{4}-\mathrm{H}\right), 9.01(1 \mathrm{H}, \mathrm{s}, \mathrm{CONH})$, $12.39(1 \mathrm{H}, \mathrm{s}$, indole $\mathrm{NH}) .{ }^{13} \mathrm{CNMR}$ (proton decoupled, DMSO-d $\left.\mathrm{d}_{6}, 150 \mathrm{MHz}\right) \delta(\mathrm{ppm}): 24.08,24.11$ (cyclopentyl $C_{3 / 4}$ ), 26.62, 32.59 (cyclopentyl $C_{2 / 5}$ ), 112.64 (ind. $C_{7}$ ), 118.53 (ind. $C_{4}$ ), 121.35 (ind. $C_{6}$ ), 126.16 (ind. $C_{3 a}$ ), 127.93 (3-Phenyl $C_{4}$ ), 128.90 (3-Phenyl $C_{3 / 5}$ ), 129.19 (ind. $C_{2}$ ), 130.19 (3-Phenyl $C_{2 / 6}$ ), 132.85 (ind. $\mathrm{C}_{5}$ ), 136.35 (3-Phenyl $\mathrm{C}_{1}$ ), 136.58 (ind. $\mathrm{C}_{7 \mathrm{a}}$ ), $156.90(\mathrm{~N}=\mathrm{C}), 167.18(\mathrm{CONH})$. Anal. Calcd. for $\mathrm{C}_{20} \mathrm{H}_{20} \mathrm{~N}_{4} \mathrm{O}_{3} \mathrm{~S}$ (396.462): C, 60.59; H, 5.08; N, 14.13; S, 8.09. Found: C, 60.73; H, 5.10; N, 13.85; S, 7.86. $\mathrm{m} / \mathrm{z}$ ESI (positive) $396.40(\mathrm{M}+\mathrm{H})^{+}$.

\subsubsection{2-[(2-Cyclohexylidenehydrazinyl)carbonyl]-3-phenyl-1H-indole-5-sulfonamide (17)}

White powder (80\%); mp 285.5-285.6 ${ }^{\circ} \mathrm{C}$; IR(KBr): v 3358, 3296, $3147(\mathrm{NH}), 1676(\mathrm{C}=\mathrm{O}) ; 1317,1149$ $(\mathrm{S}=\mathrm{O}) ;{ }^{1} \mathrm{H}$ NMR $\left(\mathrm{DMSO}_{6} / 500 \mathrm{MHz} \delta(\mathrm{ppm}): 1.34(2 \mathrm{H}, \mathrm{s},-\right.$ cyclohexyl C-H), 1.49 (2H, s, - cyclohexyl C-H), $1.60\left(4 \mathrm{H}, \mathrm{s}\right.$, -cyclohexyl C-H), $2.28\left(2 \mathrm{H}, \mathrm{s}\right.$, -cyclohexyl C-H), $7.16\left(2 \mathrm{H}, \mathrm{s}, \mathrm{SO}_{2} \mathrm{NH}_{2}\right), 7.45-7.57(5 \mathrm{H}, \mathrm{m}$, 3-phenyl C-H), $7.61\left(1 \mathrm{H}, \mathrm{d}, \mathrm{J}=8.79 \mathrm{~Hz}\right.$, indole $\left.\mathrm{C}_{7}-\mathrm{H}\right), 7.72\left(1 \mathrm{H}, \mathrm{dd}, \mathrm{J}=7.32,1.46 \mathrm{~Hz}\right.$, indole $\left.\mathrm{C}_{6}-\mathrm{H}\right), 7.94(1 \mathrm{H}$, $\mathrm{s}$, indole $\left.\mathrm{C}_{4}-\mathrm{H}\right), 9.52(1 \mathrm{H}, \mathrm{s}, \mathrm{CONH}), 12.36(1 \mathrm{H}, \mathrm{s}$, indole $\mathrm{NH}) .{ }^{13} \mathrm{CNMR}$ (proton decoupled, DMSO-d $\mathrm{d}_{6}$, $150 \mathrm{MHz}) \delta(\mathrm{ppm}): 24.80$ (cyclohexyl $\mathrm{C}_{4}$ ), 25.50, 26.26 (cyclohexyl $\left.\mathrm{C}_{3 / 5}\right), 26.56,34.69$ (cyclohexyl $\mathrm{C}_{2 / 6}$ ), 112.56 (ind. $C_{7}$ ), 118.46 (ind. $C_{4}$ ), 121.18 (ind. $C_{6}$ ), 125.95 (ind. $C_{3 a}$ ), 127.59(3-Phenyl $C_{4}$ ), 128.93 (3-Phenyl $C_{3 / 5}$ ), 129.40 (ind. $C_{2}$ ), 130.10 (3-Phenyl $C_{2 / 6}$ ), 132.82 (ind. $C_{5}$ ), 136.29 (3-Phenyl $C_{1}$ ), 136.64 
(ind. $\left.\mathrm{C}_{7 \mathrm{a}}\right), 157.26(\mathrm{~N}=\mathrm{C}), 161.59(\mathrm{CONH})$. Anal. Calcd. for $\mathrm{C}_{21} \mathrm{H}_{22} \mathrm{~N}_{4} \mathrm{O}_{3} \mathrm{~S}(410.489)$ : C, 61.44; $\mathrm{H}, 5.40 ; \mathrm{N}$, 13.65; S, 7.81. Found: C, 61.83; H, 5.17; N, 13.86; S, 7.46. m/z ESI (positive) $411.42(\mathrm{M}+\mathrm{H})^{+}$.

\subsubsection{2-\{[2-(4-Methylcyclohexylidene)hydrazinyl]carbonyl\}-3-phenyl-1H-indole-5-sulfonamide (18)}

White powder (75\%); mp 295.6-295.7 ${ }^{\circ} \mathrm{C}$; IR( $\left.\mathrm{KBr}\right)$ : v 3350, 3309, $3165(\mathrm{NH}), 1666(\mathrm{C}=\mathrm{O}) ; 1315$, $1147(\mathrm{~S}=\mathrm{O}) ;{ }^{1} \mathrm{H}$ NMR $\left(\mathrm{DMSO}_{6} / \mathrm{d}_{6} / 500 \mathrm{MHz} \delta(\mathrm{ppm}): 0.87\left(3 \mathrm{H}, \mathrm{d}, \mathrm{J}=5.37 \mathrm{~Hz}, 4-\mathrm{CH}_{3}\right), 1.04-1.14(1 \mathrm{H}, \mathrm{s}\right.$, -cyclohexyl C-H), 1.47-1.58 (4H, m, - cyclohexyl C-H), 1.72-1.80 (2H, m, -cyclohexyl C-H), 2.11-2.22 (1H, m, -cyclohexyl C-H), $2.31\left(1 \mathrm{H}, \mathrm{d}, \mathrm{J}=12.20 \mathrm{~Hz}\right.$, -cyclohexyl C-H), $7.16\left(2 \mathrm{H}, \mathrm{s}, \mathrm{SO}_{2} \mathrm{NH}_{2}\right), 7.42-7.56(5 \mathrm{H}, \mathrm{m}$, 3-phenyl C-H), $7.61\left(1 \mathrm{H}, \mathrm{d}, \mathrm{J}=8.79 \mathrm{~Hz}\right.$, indole $\left.\mathrm{C}_{7}-\mathrm{H}\right), 7.71\left(1 \mathrm{H}, \mathrm{dd}, \mathrm{J}=8.79 \mathrm{~Hz}\right.$, indole $\left.\mathrm{C}_{6}-\mathrm{H}\right), 7.93(1 \mathrm{H}, \mathrm{s}$, indole $\left.\mathrm{C}_{4}-\mathrm{H}\right), 9.51(1 \mathrm{H}, \mathrm{s}, \mathrm{CONH}), 12.35(1 \mathrm{H}$, s, indole $\mathrm{NH})$. Anal. Calcd. for $\mathrm{C}_{22} \mathrm{H}_{24} \mathrm{~N}_{4} \mathrm{O}_{3} \mathrm{~S}(424.515)$ : C, 62.24; H, 5.70; N, 13.20; S, 7.55. Found: C, 61.98; H, 5.63; N, 12.85; S, 7.16.

\subsubsection{2-\{[2-(4-Ethylcyclohexylidene)hydrazinyl]carbonyl\}-3-phenyl-1H-indole-5-sulfonamide (19)}

White powder (74\%); mp 282.4-282.5 ${ }^{\circ} \mathrm{C} ; \mathrm{IR}(\mathrm{KBr}): v$ 3348, 3311, $3176(\mathrm{NH}), 1666(\mathrm{C}=\mathrm{O}) ; 1315,1147$ $(\mathrm{S}=\mathrm{O}) ;{ }^{1} \mathrm{H}$ NMR (DMSO-d $6 / 500 \mathrm{MHz} \delta(\mathrm{ppm}): 0.85\left(3 \mathrm{H}, \mathrm{t}, \mathrm{J}=7.32 \mathrm{~Hz}, 4-\mathrm{CH}_{2} \mathrm{CH}_{3}\right), 1.16(1 \mathrm{H}, \mathrm{t}, \mathrm{J}=7.32$ $\mathrm{Hz}$, -cyclohexyl C-H), $1.21\left(2 \mathrm{H}, \mathrm{t}, \mathrm{J}=6.83 \mathrm{~Hz}, 4-\mathrm{CH}_{2} \mathrm{CH}_{3}\right), 1.35(1 \mathrm{H}$, br s, -cyclohexyl C-H), $1.49(1 \mathrm{H}$, t, J=13.67 Hz, -cyclohexyl C-H), $1.62(2 \mathrm{H}, \mathrm{d}, \mathrm{J}=12.20 \mathrm{~Hz}$, -cyclohexyl C-H), $1.75(1 \mathrm{H}, \mathrm{d}, \mathrm{J}=13.67 \mathrm{~Hz}$, -cyclohexyl C-H), $1.85(1 \mathrm{H}, \mathrm{d}, \mathrm{J}=9.27 \mathrm{~Hz}$, -cyclohexyl C-H), $2.14(1 \mathrm{H}, \mathrm{t}, \mathrm{J}=11.71 \mathrm{~Hz}$, -cyclohexyl C-H), $2.32\left(1 \mathrm{H}, \mathrm{d}, \mathrm{J}=12.20 \mathrm{~Hz}\right.$, -cyclohexyl C-H), $7.17\left(2 \mathrm{H}, \mathrm{s}, \mathrm{SO}_{2} \mathrm{NH}_{2}\right)$, 7.37-7.57 (5H, m, 3-phenyl C-H), 7.62 $\left(1 \mathrm{H}, \mathrm{d}, \mathrm{J}=8.79 \mathrm{~Hz}\right.$, indole $\left.\mathrm{C}_{7}-\mathrm{H}\right), 7.72\left(1 \mathrm{H}, \mathrm{dd}, \mathrm{J}=8.79 \mathrm{~Hz}\right.$, indole $\left.\mathrm{C}_{6}-\mathrm{H}\right), 7.93\left(1 \mathrm{H}\right.$, s, indole $\left.\mathrm{C}_{4}-\mathrm{H}\right), 9.50$ $(1 \mathrm{H}, \mathrm{s}, \mathrm{CONH}), 12.37(1 \mathrm{H}$, s, indole $\mathrm{NH})$. Anal. Calcd. for $\mathrm{C}_{23} \mathrm{H}_{24} \mathrm{~N}_{4} \mathrm{O}_{3} \mathrm{~S}(424.515)$ : C, 62.24; $\mathrm{H}, 5.70 ; \mathrm{N}$, 13.20; S, 7.55. Found: C, 62.55; H, 5.38; N, 13.63; S, 7.47.

\subsubsection{2-\{[2-(4-Propylcyclohexylidene)hydrazinyl]carbonyl\}-3-phenyl-1H-indole-5-sulfonamide (20)}

White powder (46\%); mp 249.7-250 ${ }^{\circ} \mathrm{C}$; $\mathrm{IR}(\mathrm{KBr}): v$ 3354, 3331, $3236(\mathrm{NH}), 1662(\mathrm{C}=\mathrm{O}) ; 1342,1143$ $(\mathrm{S}=\mathrm{O}) ;{ }^{1} \mathrm{H}$ NMR (DMSO-d $6 / 500 \mathrm{MHz} \delta(\mathrm{ppm}): 0.86\left(3 \mathrm{H}, \mathrm{t}, \mathrm{J}=7.32 \mathrm{~Hz}, 4-\mathrm{CH}_{2} \mathrm{CH}_{2} \mathrm{CH}_{3}\right), 1.06(1 \mathrm{H}, \mathrm{t}, \mathrm{J}=7.32$ $\mathrm{Hz}$, -cyclohexyl C-H), $1.15\left(2 \mathrm{H}, \mathrm{d}, \mathrm{J}=6.34 \mathrm{~Hz}, 4-\mathrm{CH}_{2} \mathrm{CH}_{2} \mathrm{CH}_{3}\right), 1.28\left(2 \mathrm{H}, \mathrm{q}, \mathrm{J}=7.32 \mathrm{~Hz}, 4-\mathrm{CH}_{2} \mathrm{CH}_{2} \mathrm{CH}_{3}\right)$, 1.45-1.51 (3H, m, - cyclohexyl C-H), $1.60(1 \mathrm{H}, \mathrm{d}, \mathrm{J}=12.20 \mathrm{~Hz}$, -cyclohexyl C-H), $1.74(1 \mathrm{H}, \mathrm{d}, \mathrm{J}=13.67 \mathrm{~Hz}$, -cyclohexyl C-H), $1.84(1 \mathrm{H}, \mathrm{d}, \mathrm{J}=14.00 \mathrm{~Hz}$, -cyclohexyl C-H), $2.14(1 \mathrm{H}, \mathrm{t}, \mathrm{J}=10.74 \mathrm{~Hz}$, -cyclohexyl C-H), 2.30-2.36 (1H, m, -cyclohexyl C-H), 7.16 (2H, s, $\left.\mathrm{SO}_{2} \mathrm{NH}_{2}\right)$, 7.36-7.57 $(5 \mathrm{H}, \mathrm{m}, 3$-phenyl C-H), 7.61 $(1 \mathrm{H}, \mathrm{d}$, $\mathrm{J}=8.79 \mathrm{~Hz}$, indole $\left.\mathrm{C}_{7}-\mathrm{H}\right), 7.72\left(1 \mathrm{H}, \mathrm{d}, \mathrm{J}=8.79 \mathrm{~Hz}\right.$, indole $\left.\mathrm{C}_{6}-\mathrm{H}\right), 7.93\left(1 \mathrm{H}, \mathrm{s}\right.$, indole $\left.\mathrm{C}_{4}-\mathrm{H}\right), 9.50(1 \mathrm{H}, \mathrm{s}$, $\mathrm{CONH}), 12.36(1 \mathrm{H}, \mathrm{s}$, indole $\mathrm{NH})$. Anal. Calcd. for $\mathrm{C}_{24} \mathrm{H}_{28} \mathrm{~N}_{4} \mathrm{O}_{3} \mathrm{~S}$ (452.569): C, 63.69; $\mathrm{H}, 6.24 ; \mathrm{N}, 12.38$; S, 7.09. Found: C, 63.45; H, 6.47; N, 12.78; S, 7.49.

3.1.22. 2-\{[2-(4-Phenylcyclohexylidene)hydrazinyl]carbonyl\}-3-phenyl-1H-indole-5-sulfonamide (21)

White powder (80\%); mp 312.8-313 ${ }^{\circ} \mathrm{C}$; IR(KBr): $v$ 3358, 3319, $3280(\mathrm{NH}), 1670(\mathrm{C}=\mathrm{O}) ; 1338,1159$ $(\mathrm{S}=\mathrm{O}) ;{ }^{1} \mathrm{H}$ NMR $\left(\mathrm{DMSO}^{-} \mathrm{d}_{6} / 500 \mathrm{MHz} \delta(\mathrm{ppm}): 1.28-1.41(1 \mathrm{H}, \mathrm{m}\right.$, -cyclohexyl C-H), 1.58-1.77 $(3 \mathrm{H}, \mathrm{m}$, -cyclohexyl C-H), $1.87(1 \mathrm{H}, \mathrm{d}, \mathrm{J}=13.18 \mathrm{~Hz}$, -cyclohexyl C-H), 1.96 (1H, br s, -cyclohexyl C-H), 2.26-2.39 $(1 \mathrm{H}, \mathrm{m}$, -cyclohexyl C-H), $2.46(1 \mathrm{H}, \mathrm{d}, \mathrm{J}=13.18 \mathrm{~Hz}$, -cyclohexyl C-H), $2.78(1 \mathrm{H}, \mathrm{m}$, -cyclohexyl C-H), $7.17\left(2 \mathrm{H}, \mathrm{s}, \mathrm{SO}_{2} \mathrm{NH}_{2}\right), 7.19-7.21(3 \mathrm{H}, \mathrm{m}$, cyclohexyl 4-phenyl C-H), $7.30(2 \mathrm{H}, \mathrm{t}, \mathrm{J}=7.81 \mathrm{~Hz}$, cyclohexyl 4-phenyl C-H), 7.42-7.48 (1H, m, 3-phenyl C-H), 7.49-7.55 (4H, m, 3-phenyl C-H), 7.63 (1H, d, J=8.79 $\mathrm{Hz}$, indole $\left.\mathrm{C}_{7}-\mathrm{H}\right), 7.71\left(1 \mathrm{H}, \mathrm{dd}, \mathrm{J}=8.79,0.98 \mathrm{~Hz}\right.$, indole $\left.\mathrm{C}_{6}-\mathrm{H}\right), 7.95\left(1 \mathrm{H}, \mathrm{s}\right.$, indole $\left.\mathrm{C}_{4}-\mathrm{H}\right), 9.61(1 \mathrm{H}, \mathrm{s}$, $\mathrm{CONH}), 12.37(1 \mathrm{H}$, s, indole $\mathrm{NH})$. Anal. Calcd. for $\mathrm{C}_{27} \mathrm{H}_{26} \mathrm{~N}_{4} \mathrm{O}_{3} \mathrm{~S}$ (486.585): C, 66.65; H, 5.39; N, 11.51; S, 6.59. Found: C, 66.38; H, 5.57; N, 11.88; S, 8.94.

3.1.23. 2-\{[2-(4-tert-buthylcyclohexylidene)hydrazinyl]carbonyl\}-3-phenyl-1H-indole-5-sulfonamide (22)

White powder (55\%); mp 284.9-285.2 ${ }^{\circ} \mathrm{C}$; IR(KBr): v 3358, 3284, $3161(\mathrm{NH}), 1662(\mathrm{C}=\mathrm{O}) ; 1342,1143$ $(\mathrm{S}=\mathrm{O}) ;{ }^{1} \mathrm{H}$ NMR (DMSO-d 6 /500 MHz $\delta$ (ppm): $0.83\left(9 \mathrm{H}, \mathrm{s}, 3 \times \mathrm{CH}_{3}\right), 1.10-1.23(3 \mathrm{H}, \mathrm{m},-$ cyclohexyl C-H), $1.45(1 \mathrm{H}, \mathrm{td}, \mathrm{J}=14.15,4.88 \mathrm{~Hz}$, -cyclohexyl C-H), $1.64(1 \mathrm{H}, \mathrm{d}, \mathrm{J}=10.74 \mathrm{~Hz}$, -cyclohexyl C-H), 1.80-1.89 
(2H, m, -cyclohexyl C-H), $2.13(1 \mathrm{H}, \mathrm{t}, \mathrm{J}=10.74 \mathrm{~Hz}$, -cyclohexyl C-H), 2.36 (1H, d, J=12.69 Hz, -cyclohexyl C-H), $7.16\left(2 \mathrm{H}, \mathrm{s}, \mathrm{SO}_{2} \mathrm{NH}_{2}\right), 7.47-7.56\left(5 \mathrm{H}, \mathrm{m}, 3\right.$-phenyl C-H), $7.61\left(1 \mathrm{H}, \mathrm{d}, \mathrm{J}=8.79 \mathrm{~Hz}\right.$, indole $\left.\mathrm{C}_{7}-\mathrm{H}\right), 7.71$ $\left(1 \mathrm{H}, \mathrm{d}, \mathrm{J}=8.79 \mathrm{~Hz}\right.$, indole $\left.\mathrm{C}_{6}-\mathrm{H}\right), 7.94\left(1 \mathrm{H}\right.$, s, indole $\left.\mathrm{C}_{4}-\mathrm{H}\right), 9.49(1 \mathrm{H}, \mathrm{s}, \mathrm{CONH}), 12.36(1 \mathrm{H}$, s, indole $\mathrm{NH})$. Anal. Calcd. for $\mathrm{C}_{25} \mathrm{H}_{30} \mathrm{~N}_{4} \mathrm{O}_{3} \mathrm{~S}$ (466.595): $\mathrm{C}, 64.35 ; \mathrm{H}, 6.48 ; \mathrm{N}, 12.01 ; \mathrm{S}, 6.87$. Found: $\mathrm{C}, 64.28 ; \mathrm{H}, 6.35 ; \mathrm{N}$, $11.90 ; \mathrm{S}, 6.47$.

\subsubsection{2-\{[2-(1-Methylpiperidine-4-ylidene)hydrazinyl]carbonyl\}-3-phenyl-1H-indole-5-sulfonamide (23)}

White powder (65\%); mp 250.1-250.3 ${ }^{\circ} \mathrm{C}$; IR(KBr): v 3352, 3334, $3305(\mathrm{NH}), 1666(\mathrm{C}=\mathrm{O}) ; 1315,1159$ $(\mathrm{S}=\mathrm{O}) ;{ }^{1} \mathrm{H}$ NMR (DMSO- $\mathrm{d}_{6} / 500 \mathrm{MHz} \delta(\mathrm{ppm}): 1.71\left(2 \mathrm{H}, \mathrm{br} \mathrm{s}\right.$, piperidine $\left.\mathrm{C}_{3 / 5}-\mathrm{H}\right), 2.17\left(5 \mathrm{H}, \mathrm{br} \mathrm{s}, \mathrm{N}-\mathrm{CH}_{3}\right.$ and piperidine $\left.\mathrm{C}_{2 / 6}-\mathrm{H}\right), 2.30\left(2 \mathrm{H}\right.$, br s, piperidine $\left.\mathrm{C}_{3 / 5}-\mathrm{H}\right), 2.42\left(2 \mathrm{H}\right.$, br s, piperidine $\left.\mathrm{C}_{2 / 6}-\mathrm{H}\right), 7.17(2 \mathrm{H}$, s, $\left.\mathrm{SO}_{2} \mathrm{NH}_{2}\right), 7.38-7.54\left(5 \mathrm{H}, \mathrm{m}, 3-\right.$ phenyl C-H), $7.61\left(1 \mathrm{H}, \mathrm{d}, \mathrm{J}=8.79 \mathrm{~Hz}\right.$, indole $\left.\mathrm{C}_{7}-\mathrm{H}\right), 7.72(1 \mathrm{H}, \mathrm{dd}, \mathrm{J}=8.78$, $1.46 \mathrm{~Hz}$, indole $\left.\mathrm{C}_{6}-\mathrm{H}\right), 7.96\left(1 \mathrm{H}\right.$, s, indole $\left.\mathrm{C}_{4}-\mathrm{H}\right), 9.66(1 \mathrm{H}, \mathrm{s}, \mathrm{CONH}), 12.39(1 \mathrm{H}$, s, indole $\mathrm{NH}) .{ }^{13} \mathrm{CNMR}$ (proton decoupled, $\left.\mathrm{DMSO}_{\mathrm{d}}, 150 \mathrm{MHz}\right) \delta(\mathrm{ppm}): 26.42,33.99$ (piperidine $\left.\mathrm{C}_{3 / 5}\right), 45.20\left(\mathrm{~N}^{-} \mathrm{CH}_{3}\right), 53.88$, 55.29 (piperidine $C_{2 / 6}$ ), 112.66 (ind. $C_{7}$ ), 118.52 (ind. $C_{4}$ ), 118.60 (ind. $C_{3}$ ), 121.28 (ind. $C_{6}$ ), 125.86 (ind. $C_{3 a}$ ), 127.59 (3-Phenyl $C_{4}$ ), 128.93 (3-Phenyl $C_{3 / 5}$ ), 132.93 (ind. $C_{5}$ ), 130.19 (3-Phenyl $C_{2 / 6}$ ), 136.36 (3-Phenyl $\mathrm{C}_{1}$ ), 136.64 (ind. $\mathrm{C}_{7 \mathrm{a}}$ ), $157.51(\mathrm{~N}=\mathrm{C}), 158.57(\mathrm{CONH}) .{ }^{13} \mathrm{C}-\mathrm{NMR}$ (HSQC-2D, DMSO-d ${ }_{6}, 125$ $\mathrm{MHz}) \delta(\mathrm{ppm}): 26.40$ [piperidine $\mathrm{C}_{3 / 5}-1.71\left(2 \mathrm{H}, \mathrm{br} \mathrm{s}\right.$, piperidine $\left.\mathrm{C}_{3 / 5}-\mathrm{H}\right)$ ], 33.95 [piperidine $\mathrm{C}_{3 / 5}-2.30$ $\left(2 \mathrm{H}\right.$, br s, piperidine $\left.\left.\mathrm{C}_{3 / 5}-\mathrm{H}\right)\right], 45.20$ [N- $\mathrm{CH}_{3}-2.17\left(5 \mathrm{H}\right.$, br s, $\mathrm{N}^{-} \mathrm{CH}_{3}$ and piperidine $\left.\left.\mathrm{C}_{2 / 6}-\mathrm{H}\right)\right], 53.87$ [piperidine $\mathrm{C}_{2 / 6}-2.17\left(5 \mathrm{H}, \mathrm{br} \mathrm{s}, \mathrm{N}-\mathrm{CH}_{3}\right.$ and piperidine $\left.\mathrm{C}_{2 / 6}-\mathrm{H}\right)$ ], 55.28 [piperidine $\mathrm{C}_{2 / 6}-2.42(2 \mathrm{H}$, br s, piperidine $\left.\mathrm{C}_{2 / 6}-\mathrm{H}\right)$ ], 112.63 [ind. $\mathrm{C}_{7}-7.61\left(1 \mathrm{H}, \mathrm{d}, \mathrm{J}=8.79 \mathrm{~Hz}\right.$, indole $\left.\mathrm{C}_{7}-\mathrm{H}\right)$ ], 118.55 [ind $\mathrm{C}_{4}-7.96(1 \mathrm{H}$, $\mathrm{s}$, indole $\left.\mathrm{C}_{4}-\mathrm{H}\right)$ ], 121.27 [ind. $\mathrm{C}_{6}-7.72\left(1 \mathrm{H}, \mathrm{dd}, \mathrm{J}=8.78,1.46 \mathrm{~Hz}\right.$, indole $\left.\mathrm{C}_{6}-\mathrm{H}\right)$ ], 127.58 [3-phenyl $\mathrm{C}_{4}$ - 7.38-7.54 (5H, m, 3-phenyl C-H)], 128.92 [3-phenyl C $3 / 5$ - 7.38-7.54 (5H, m, 3-phenyl C-H)], 130.14 [3-phenyl $\mathrm{C}_{2 / 6}-7.38-7.54\left(5 \mathrm{H}, \mathrm{m}, 3\right.$-phenyl C-H)]. Anal. Calcd. for $\mathrm{C}_{21} \mathrm{H}_{23} \mathrm{~N}_{5} \mathrm{O}_{3} \mathrm{~S}$ (425.504): C, 59.28; $\mathrm{H}, 5.45 ; \mathrm{N}, 16.46$; S, 7.54. Found: C, 58.87; H, 5.30; N, 16.20; S, 7.36.

\subsubsection{2-\{[2-(1-Benzylpiperidine-4-ylidene)hydrazinyl]carbonyl\}-3-phenyl-1H-indole-5-sulfonamide (24)}

White powder (70\%); mp 251.2-251.4 ${ }^{\circ} \mathrm{C}$; IR(KBr): $v$ 3379, 3352, $3265(\mathrm{NH}), 1685(\mathrm{C}=\mathrm{O}) ; 1311$, $1176(\mathrm{~S}=\mathrm{O}) ;{ }^{1} \mathrm{H}$ NMR (DMSO-d 6 /500 MHz $\delta$ (ppm): $1.73(2 \mathrm{H}$, br s, piperidine $\mathrm{C}-\mathrm{H}), 2.26-2.31(4 \mathrm{H}, \mathrm{m}$, piperidine $\mathrm{C}-\mathrm{H}), 2.48(2 \mathrm{H}$, s, piperidine $\mathrm{C}-\mathrm{H}), 3.37(2 \mathrm{H}, \mathrm{s}, \mathrm{N}-\mathrm{CH} 2-), 7.16\left(2 \mathrm{H}, \mathrm{s}, \mathrm{SO}_{2} \mathrm{NH}_{2}\right), 7.25-7.35$ $(5 \mathrm{H}, \mathrm{m}$, benzyl C-H), 7.39-7.45 $(1 \mathrm{H}, \mathrm{m}, 3$-phenyl C-H), 7.49-7.55 $(4 \mathrm{H}, \mathrm{m}, 3$-phenyl C-H), $7.61(1 \mathrm{H}, \mathrm{d}$, $\mathrm{J}=8.30 \mathrm{~Hz}$, indole $\left.\mathrm{C}_{7}-\mathrm{H}\right), 7.72\left(1 \mathrm{H}, \mathrm{d}, \mathrm{J}=8.79 \mathrm{~Hz}\right.$, indole $\left.\mathrm{C}_{6}-\mathrm{H}\right), 7.96\left(1 \mathrm{H}\right.$, s, indole $\left.\mathrm{C}_{4}-\mathrm{H}\right), 9.67(1 \mathrm{H}, \mathrm{s}$, $\mathrm{CONH}), 12.37\left(1 \mathrm{H}, \mathrm{s}\right.$, indole NH). ${ }^{13} \mathrm{CNMR}$ (proton decoupled, DMSO-d $\left.\mathrm{d}_{6}, 150 \mathrm{MHz}\right) \delta$ (ppm): 26.42, 33.93 (piperidine $\left.\mathrm{C}_{3 / 5}\right), 51.50,52.72$ (piperidine $\mathrm{C}_{2 / 6}$ ), $61.01\left(\mathrm{~N}_{-} \mathrm{CH}_{2}-\mathrm{Ph}\right.$ ), 112.53 (ind. $\mathrm{C}_{7}$ ), 118.45 (ind. $\left.\mathrm{C}_{4}\right), 122.49$ (ind. $\left.\mathrm{C}_{6}\right), 125.76$ (ind. $\left.\mathrm{C}_{3 \mathrm{a}}\right), 126.84,127.40\left(2 \times\right.$ Phenyl $\left.\mathrm{C}_{4}\right), 128.08,128.61\left(2 \times\right.$ Phenyl $\left._{3 / 5}\right)$, 128.82 (ind. $\left.C_{2}\right), 129.38,130.08$ ( 2 x Phenyl $\left.C_{2 / 6}\right), 132.90$ (ind. $\left.C_{5}\right), 136.30,136.57$ ( 2 x Phenyl $\left.C_{1}\right), 138.30$ (ind. $\left.\mathrm{C}_{7 \mathrm{a}}\right), 157.50(\mathrm{~N}=\mathrm{C}), 158.50(\mathrm{CONH})$. Anal. Calcd. for $\mathrm{C}_{27} \mathrm{H}_{27} \mathrm{~N}_{5} \mathrm{O}_{3} \mathrm{~S}$ (501.599): $\mathrm{C}, 64.65 ; \mathrm{H}, 5.43 ; \mathrm{N}$, 13.96; $\mathrm{S}, 6.39$. Found: $\mathrm{C}, 64.87 ; \mathrm{H}, 5.20 ; \mathrm{N}, 14.22 ; \mathrm{S}, 6.16$. m/z ESI (positive) $502.63(\mathrm{M}+\mathrm{H})^{+}$.

\subsection{Enzyme Inhibition Studies}

A stopped-flow instrument (SX.18 MV-R Applied Photophysics model) was used for assaying the $\mathrm{CO}_{2}$ hydration activity of various $\mathrm{CA}$ isozymes, as reported by Khalifah [29]. Phenol red (at a concentration of $0.2 \mathrm{mM}$ ) was used as indicator, working at the absorbance maximum of $557 \mathrm{~nm}$ with $10 \mathrm{mM}$ Hepes (pH 7.4) as a buffer and $0.1 \mathrm{M} \mathrm{NaClO}_{4}$ (for constantly maintaining the ionic strength; this anion was not inhibitory in the used concentration) following the $\mathrm{CA}$-catalyzed $\mathrm{CO}_{2}$ hydration reaction for a period of 5-10 s. Saturated $\mathrm{CO}_{2}$ solutions in water at $25^{\circ} \mathrm{C}$ were used as substrates. Stock solutions of inhibitors were prepared at a concentration of $10 \mathrm{mM}$ (in DMSO/water 1:1, v/v) and dilutions up to $0.01 \mathrm{nM}$ were done with the assay buffer mentioned above. At least seven different inhibitor concentrations were used for measuring the inhibition constant. For allowing the complete formation of the enzyme-inhibitor adduct, the inhibitor and the enzyme were pre-incubated for $15 \mathrm{~min}$. $\mathrm{IC}_{50}$ values were obtained from dose response curves working at seven different concentrations of 
the test compound (from $0.1 \mathrm{nM}$ to $50 \mathrm{mM}$ ) by fitting the curves using PRISM (www.graphpad.com) and non-linear least squares methods, the obtained values representing the mean of at least three different determinations. The inhibition constants $\left(K_{\mathrm{I}}\right)$ were derived from the $\mathrm{IC}_{50}$ values by using the Cheng-Prusoff equation as follows: $K_{\mathrm{I}}=\mathrm{IC}_{50} /\left(1+[\mathrm{S}] / K_{\mathrm{M}}\right)$ where [S] represents the $\mathrm{CO}_{2}$ concentration at which the measurement was carried out, and $K_{\mathbf{M}}$ represents the concentration of the substrate at which the enzyme activity was at half maximal. As reported earlier, all enzymes used were recombinant and produced in Escherichia Coli. [30-33]. The concentrations of enzymes used in the assay were: hCA I, $11.9 \mathrm{nM}$; hCA II, $7.7 \mathrm{nM}$; hCA IX, $9.1 \mathrm{nM}$, and hCA XII, $11.5 \mathrm{nM}$.

\subsection{Molecular Modeling Studies}

The three-dimensional structures of all ligands were prepared in their lowest energy conformation using the MOE software package (v2018.0101, Chemical Computing Group, Inc, Montreal, Canada). The sulfonamide nitrogen atoms were assigned a negative charge $\left(\mathrm{R}-\mathrm{SO}_{2} \mathrm{NH}-\right)$, and the ligands were energy minimized (MMFF94x force field).

All protein structures were obtained from the RCSB protein databank: hCA I in complex with topiramate (pdb: 3lxe, $1.90 \AA$ ), hCA II in complex with water (pdb: 4e3d, $1.60 \AA$ ), hCA IX in complex with acetazolamide (pdb: 3iai; $2.20 \AA$ ), and hCA XII in complex with acetazolamide (pdb: 1jd0; $1.50 \AA$ ). The protein atoms and the active site zinc ions were retained, and all other atoms were omitted. The remaining structure was protonated using the protonate $3 \mathrm{D}$ functionality of $\mathrm{MOE}$, and subsequently, the obtained structure was energy-minimized (AMBER14:EHT) [34]. Finally, the obtained protein models were superposed on the hCA I structure using the backbone C $\alpha$-atoms and all $\mathrm{Zn}^{2+-}$ ions, zinc-binding histidines, and the overall backbone atoms superposed well [root-mean-square deviation (RMSD) value: $1.281 \AA$ ] .

Docking calculations were performed using the FlexX docking tool (v2.3.2; BioSolveIT GmbH, St. Augustin, Germany) within MOE. The binding pocket was defined as all residues within $6.5 \AA$ of the reference ligand, acetazolamide. The sulfonamide tail of the ligands was forced to adopt a similar orientation and interactions to the $\mathrm{Zn}^{2+-}$ ion as observed for acetazolamide using a pharmacophore model. All ligands were docked fifty times, and the best scoring three poses were subjected to refinement calculations. To this end, the ligand and the binding pocket residues were energy minimized and rescored using a GBVI/WSA force field [35].

\section{Conclusions}

A total of 21 new indole-based sulfonamide derivatives were synthesized and tested against the tumor-associated hCA IX and XII and the widespread off-targets, hCA I and II. The compounds showed potent inhibition in the lower nanomolar range and at least $\sim$ six-fold selectivity for hCA IX and XII over hCA I and II. Molecular modeling studies were subsequently performed to gather insight in the possible binding modes between the newly developed CAIs and their respective target hCAs. This information may result in more potent and more selective CAIs for hCA IX and XII.

Author Contributions: Conceptualization, Ö.G.A. and C.T.S.; Synthesis, K.D.Y., N.M.A. and Ö.G.A., Molecular modeling, A.A. and K.D.Y.; Enzyme inhibition assays: S.B., C.T.S. Writing-Original Draft Preparation, K.D.Y., A.A. Ö.G.A.; Writing-Review \& Editing, A.A. and Ö.G.A.; Supervision, Ö.G.A.; Project Administration, Ö.G.A.; Funding Acquisition, Ö.G.A.

Funding: This research was funding by the Istanbul University Scientific Research Projects Department under project numbers TSA-2019-30535.

Conflicts of Interest: The authors declare no conflict of interest. The funders had no role in the design of the study; in the collection, analyses, or interpretation of data; in the writing of the manuscript, or in the decision to publish the results. 


\section{Abbreviations}

CA Carbonic Anhydrases

hCA Human Carbonic Anhydrases

CAI Carbonic Anhydrase Inhibitor

\section{References}

1. Supuran, C.T. Carbonic anhydrase inhibitors and activators for novel therapeutic applications. Future Med. Chem. 2011, 3, 1165-1180. [CrossRef] [PubMed]

2. Supuran, C.T. Carbonic anhydrases: Novel therapeutic applications for inhibitors and activators. Nat. Rev. Drug Discov. 2008, 7, 168-181. [CrossRef] [PubMed]

3. Supuran, C.T.; Scozzafava, A. Carbonic anhydrase inhibitors and their therapeutic potential. Expert Opin. Ther. Pat. 2005, 10, 575-600. [CrossRef]

4. Neri, D.; Supuran, C.T. Interfering with $\mathrm{pH}$ regulation in tumors as a therapeutic strategy. Nat. Rev. Drug Discov. 2011, 10, 767-777. [CrossRef]

5. Akdemir, A.; Güzel-Akdemir, Ö. The Structure, Physiological Role, and Potential Medicinal Applications of Carbonic Anhydrase V. In Carbonic Anhydrases as Biocatalysts: From Theory to Medical and Industrial Applications; Elsevier: Amsterdam, The Netherlands, 2015; pp. 125-138. ISBN 9780444632630.

6. Supuran, C.T.; Scozzafava, A. Carbonic anhydrases as targets for medicinal chemistry. Bioorganic Med. Chem. 2007, 15, 4336-4350. [CrossRef] [PubMed]

7. Supuran, C.T. Structure and function of carbonic anhydrases. Biochem. J. 2016, 473, 2023-2032. [CrossRef] [PubMed]

8. Supuran, C.T.; Briganti, F.; Tilli, S.; Chegwidden, W.R.; Scozzafava, A. Carbonic anhydrase inhibitors: Sulfonamides as antitumor agents? Bioorganic Med. Chem. 2001, 9, 703-714. [CrossRef]

9. Carta, F.; Supuran, C.T. Diuretics with carbonic anhydrase inhibitory action: A patent and literature review (2005-2013). Expert Opin. Ther. Pat. 2013, 23, 681-691. [CrossRef]

10. Masini, E.; Carta, F.; Scozzafava, A.; Supuran, C.T. Antiglaucoma carbonic anhydrase inhibitors: A patent review. Expert Opin. Ther. Pat. 2013, 23, 705-716. [CrossRef] [PubMed]

11. De Simone, G.; Scozzafava, A.; Supuran, C.T. Which carbonic anhydrases are targeted by the antiepileptic sulfonamides and sulfamates? Chem. Biol. Drug Des. 2009, 74, 317-321. [CrossRef] [PubMed]

12. Akdemir, A.; Güzel-Akdemir, Ö.; Scozzafava, A.; Capasso, C.; Supuran, C.T. Inhibition of tumor-associated human carbonic anhydrase isozymes IX and XII by a new class of substituted-phenylacetamido aromatic sulfonamides. Bioorganic Med. Chem. 2013, 21, 5228-5232. [CrossRef]

13. Supuran, C.T. Carbonic Anhydrase Inhibition and the Management of Hypoxic Tumors. Metabolites 2017, 7, 48. [CrossRef]

14. Nocentini, A.; Supuran, C.T. Carbonic anhydrase inhibitors as antitumor/antimetastatic agents: A patent review (2008-2018). Expert Opin. Ther. Pat. 2018, 28, 729-740. [CrossRef]

15. Puccetti, L.; Fasolis, G.; Vullo, D.; Chohan, Z.H.; Scozzafava, A.; Supuran, C.T. Carbonic anhydrase inhibitors. Inhibition of cytosolic/tumor-associated carbonic anhydrase isozymes I, II, IX, and XII with Schiff's bases incorporating chromone and aromatic sulfonamide moieties, and their zinc complexes. Bioorganic Med. Chem. Lett. 2005, 15, 3096-3101. [CrossRef] [PubMed]

16. Supuran, C.T. How many carbonic anhydrase inhibition mechanisms exist? J. Enzyme Inhib. Med. Chem. 2016, 31, 345-360. [PubMed]

17. Supuran, C.T. Advances in structure-based drug discovery of carbonic anhydrase inhibitors. Expert Opin. Drug Discov. 2017, 12, 61-88. [CrossRef] [PubMed]

18. Vullo, D.; Scozzafava, A.; T. Supuran, C.; Guzela, O.; Innocenti, A. 3-Phenyl-1H-Indole-5-Sulfonamides: Structure-Based Drug Design of a Promising Class of Carbonic Anhydrase Inhibitors. Curr. Pharm. Des. 2010, 16, 3317-3326.

19. Güzel, Ö.; Innocenti, A.; Scozzafava, A.; Salman, A.; Parkkila, S.; Hilvo, M.; Supuran, C.T. Carbonic anhydrase inhibitors: Synthesis and inhibition studies against mammalian isoforms I-XV with a series of 2-(hydrazinocarbonyl)-3-substituted-phenyl-1H-indole-5-sulfonamides. Bioorganic Med. Chem. 2008, 16, 9113-9120. [CrossRef] 
20. Güzel, Ö.; Temperini, C.; Innocenti, A.; Scozzafava, A.; Salman, A.; Supuran, C.T. Carbonic anhydrase inhibitors. Interaction of 2-(hydrazinocarbonyl)-3-phenyl-1H-indole-5-sulfonamide with 12 mammalian isoforms: Kinetic and X-ray crystallographic studies. Bioorganic Med. Chem. Lett. 2008, 18, 152-158. [CrossRef]

21. Ergenç, N.; Salman, A.; Gürsoy, A.; Pharmazie, G. Synthesis and antifungal evaluation of some 3-phenyl-2, 5-disubstituted indoles derived from new ethyl-2-benzyl-2-[N-(aryl)hydrazono] ethanoates. Die Pharm. 1990, 45, 346-347. [CrossRef]

22. Ergenç, N.; Günay, N.S.; Demirdamar, R. Synthesis and antidepressant evaluation of new 3-phenyl-5-sulfonamidoindole derivatives. Eur. J. Med. Chem. 1998, 33, 143-148. [CrossRef]

23. Kazius, J.; Mcguire, R.; Bursi, R. Derivation and Validation of Toxicophores for Mutagenicity Prediction. J. Med. Chem. 2005, 48, 312-320. [CrossRef]

24. TI, O. Property distribution of drug-related chemical databases. J. Comput. Aided. Mol. Des. 2000, 14, 251-264.

25. Tetko, I.V.; Tanchuk, V.Y. Application of Associative Neural Networks for Prediction of Lipophilicity in ALOGPS 2.1 Program. J. Chem. Inf. Comput. Sci. 2002, 42, 1136-1145. [CrossRef]

26. Tetko, I.V.; Gasteiger, J.; Todeschini, R.; Mauri, A.; Livingstone, D.; Ertl, P.; Palyulin, V.A.; Radchenko, E.V.; Zefirov, N.S.; Makarenko, A.S.; et al. Virtual computational chemistry laboratory-Design and description. J. Comput. Aided. Mol. Des. 2005, 19, 453-463. [CrossRef]

27. Christopher, A.L. Drug-like properties and the causes of poor solubility and poor permeability. J. Pharmacol. Toxicol. Methods 2000, 44, 235-249.

28. Lipinski, C.A.; Lombardo, F.; Dominy, B.W.; Feeney, P.J. Experimental and computational approaches to estimate solubility and permeability in drug discovery and development settings. Adv. Drug Deliv. Rev. 2012, 64, 4-17. [CrossRef]

29. Khalifah, R.G. The Carbon Dioxide Hydration Activity of Carbonic Anhydrase. J. Biol. Chem. 1971, 246, 2561-2573. [PubMed]

30. Lou, Y.; McDonald, P.C.; Oloumi, A.; Chia, S.; Ostlund, C.; Ahmadi, A.; Kyle, A.; Auf Dem Keller, U.; Leung, S.; Huntsman, D.; et al. Targeting tumor hypoxia: Suppression of breast tumor growth and metastasis by novel carbonic anhydrase IX inhibitors. Cancer Res. 2011, 71, 3364-3376. [CrossRef] [PubMed]

31. Casey, J.R.; Morgan, P.E.; Vullo, D.; Scozzafava, A.; Mastrolorenzo, A.; Supuran, C.T. Carbonic Anhydrase Inhibitors. Design of Selective, Membrane-Impermeant Inhibitors Targeting the Human Tumor-Associated Isozyme IX. J. Med. Chem. 2004, 47, 2337-2347. [CrossRef] [PubMed]

32. Tars, K.; Vullo, D.; Kazaks, A.; Leitans, J.; Lends, A.; Grandane, A.; Zalubovskis, R.; Scozzafava, A.; Supuran, C.T. Sulfocoumarins (1,2-benzoxathiine-2,2-dioxides): A class of potent and isoform-selective inhibitors of tumor-associated carbonic anhydrases. J. Med. Chem. 2013, 56, 293-300. [CrossRef] [PubMed]

33. Supuran, C.T. Carbon- versus sulphur-based zinc binding groups for carbonic anhydrase inhibitors? J. Enzyme Inhib. Med. Chem. 2018, 33, 485-495. [CrossRef] [PubMed]

34. Labute, P. Protonate3D: Assignment of ionization states and hydrogen coordinates to macromolecular structures. Proteins Struct. Funct. Bioinforma. 2009, 75, 187-205. [CrossRef] [PubMed]

35. Labute, P. The generalized born/volume integral implicit solvent model: Estimation of the free energy of hydration using London dispersion instead of atomic surface area. J. Comput. Chem. 2008, 29, 1693-1698. [CrossRef] [PubMed]

(C) 2019 by the authors. Licensee MDPI, Basel, Switzerland. This article is an open access article distributed under the terms and conditions of the Creative Commons Attribution (CC BY) license (http://creativecommons.org/licenses/by/4.0/). 